\title{
Low efficiency upconversion nanoparticles for high-resolution coalignment of near-infrared and visible light paths on a light microscope
}

\author{
Sriramkumar Sundaramoorthy ${ }^{1}$, Adrian Garcia Badaracco ${ }^{2}$, Sophia M. Hirsch ${ }^{3}$, Jun Hong \\ Park $^{2}$, Tim Davies ${ }^{1}$, Julien Dumont ${ }^{4}$, Mimi Shirasu-Hiza ${ }^{3}$, Andrew C. Kummel ${ }^{2}$, and Julie C. \\ Canman ${ }^{1, *}$ \\ ${ }^{1}$ Columbia University Medical Center, Dept. of Pathology and Cell Biology, New York, NY 10032 \\ ${ }^{2}$ University of California, San Diego, Section of Chemical and Materials Science, La Jolla, CA \\ 92093 \\ ${ }^{3}$ University Medical Center, Dept. of Genetics and Development, New York, NY 10032 \\ ${ }^{4}$ Institut Jacques Monod, CNRS, UMR 7592, University Paris Diderot, Sorbonne Paris Cité \\ F-75205 Paris, France
}

\section{Abstract}

The combination of near infrared (NIR) and visible wavelengths in light microscopy for biological studies is increasingly common. For example, many fields of biology are developing the use of NIR for optogenetics, in which an NIR laser induces a change in gene expression and/or protein function. One major technical barrier in working with both NIR and visible light on an optical microscope is obtaining their precise coalignment at the imaging plane position. Photon upconverting particles (UCPs) can bridge this gap as they are excited by NIR light but emit in the visible range via an anti-Stokes luminescence mechanism. Here, two different UCPs have been identified, high-efficiency micro ${ }^{540}$-UCPs and lower efficiency nano ${ }^{545}$-UCPs, that respond to NIR light and emit visible light with high photostability even at very high NIR power densities (>25,000 Suns). Both of these UCPs can be rapidly and reversibly excited by visible and NIR light and emit light at visible wavelengths detectable with standard emission settings used for Green Fluorescent Protein (GFP), a commonly used genetically-encoded fluorophore. However, the high efficiency micro ${ }^{540}$-UCPs were suboptimal for NIR and visible light coalignment, due to their larger size and spatial broadening from particle-to-particle energy transfer consistent with a long lived excited state and saturated power dependence. In contrast, the lower efficiency nano-UCPs were superior for precise coalignment of the NIR beam with the visible light path $(\sim 2 \mu \mathrm{m}$ versus $\sim 8 \mu \mathrm{m}$ beam broadening respectively) consistent with limited particle-to-particle energy transfer,

*Corresponding author: Julie C. Canman, 630 W. $168^{\text {th }}$ St., New York, NY 10032, (212) 305-5017, jcc2210@ cumc.columbia.edu. Supplemental material

The supplemental material contains supplemental Figures S1-S5 and supplemental figure legends.

Author contributions

JCC, SS, and TD conceived of the experiments. SS conducted all of the experiments unless noted. JHP conducted the AFM and size distribution analysis. AGB conducted SEM, EDS, and beam fit analysis and efficiency calculations. SMH conducted the IR-LEGO experiments. JCC, SS, ACK, MSH, AGB, JHP, SMH, and JD made intellectual contributions and wrote the manuscript. JCC, SMH, and SS constructed the figures. 
superlinear power dependence for emission, and much smaller particle size. Furthermore, the nano-UCPs were superior to a traditional two-camera method for NIR and visible light path alignment in an in vivo Infrared-Laser-Evoked Gene Operator (IR-LEGO) optogenetics assay in the budding yeast $S$. cerevisiae. In summary, nano-UCPs are powerful new tools for coaligning NIR and visible light paths on a light microscope.

\section{Keywords}

Infrared laser; visible light; microscope; upconverter; upconverting particle; optogenetics; laser alignment; light-path coalignment

\section{Introduction}

The use of near infrared wavelengths (NIR) as a tool in molecular and biological experiments on the light microscope is on the rise. Multi-photon imaging, optical tweezers, and laser-evoked gene control approaches all use NIR lasers for in vitro and in vivo imaging, particle trapping, single molecule studies, and cell-specific induction of gene expression ${ }^{1-9}$. A recent area of development is the use of NIR light in optogenetics studies. High-power spatially and temporally targeted NIR light is used directly or indirectly (via upconversion) to regulate light-sensitive proteins and/or heat-sensitive gene promoters in a cellular or subcellular region of interest. In optogenetics experiments, targeted NIR light is often continually focused on a cellular or sub-cellular region throughout an experiment for up to several hours to produce the desired change in protein function or gene expression. In most optogenetics systems, fluorescently-tagged reporters are used to simultaneously monitor the in vivo effects of NIR illumination on the biological specimen over time on the same microscope $\mathrm{e}^{10-17}$. For these studies, the NIR light must be both precisely targeted to $\mu \mathrm{m}$ scale structures and focused at the imaging plane so the optogenetic effect can be simultaneously monitored in the same plane. Thus, precision and repeatability in NIR-based optogenetics experiments depends on accurate and reliable coalignment of NIR and visible light in the X-, Y-, and Z-dimensions at the imaging plane of the microscope. NIR light is invisible to the human eye, and most microscope optics are not chromatically corrected for NIR wavelengths, further complicating coalignment in the Z-dimension.

Currently, NIR-laser and visible light coalignment on the light microscope relies on having an NIR-sensitive camera that is parfocal with the low-light sensitive camera used for timelapse biological imaging (Figure S5). Most commonly, small particles and/or a patterned mirrored slide are used as a specimen to co-align visible light and the peak NIR laser intensity using an inexpensive visible light sensitive camera that is also sensitive to highpower NIR light or an expensive NIR-sensitive camera. Coalignment can also be accomplished using the NIR laser to optically trap a sub-micron-scale bead and then moving the trapped bead to the visible light imaging plane. All of these methods depend on a split light path to two different cameras, one of which is sensitive to NIR light (or bead position) and another for low light fluorescence time-lapse imaging (e.g. ${ }^{2,18}$ ). The indirect and dual camera/detector nature of current alignment techniques also requires complex image/camera 
positional transfer algorithms and generally makes it difficult to obtain precise parfocality between the NIR laser light path and the visible light path at high resolution in X, Y, and Z.

Photon upconverting particles are a one-step, single camera tool to facilitate precise parfocality between NIR laser light path and fluorescence emission light at the imaging plane (Figure S5). Photon upconverting (or upconverter) particles (UCPs) are inorganic crystals doped with rare earth elements. They are commonly used in security ${ }^{19}$, solar cell development ${ }^{20}$, and, more recently in light microscopy as "fluorophores" to biochemically tag proteins of interest (e.g. with antibody or biotin linkage) ${ }^{21-26}$. Upconverting nanoparticles absorb two, three, or more photons of NIR light and emit higher energy wavelengths of light within the visible range via an anti-Stokes luminescence mechanism with varying efficiency ${ }^{21,27}$. We first tested high efficiency micro ${ }^{540}-\mathrm{UCPs}^{28}$ with a standard filter set for use with Green Fluorescent Protein (GFP; 525/50 or 525 $\pm 25 \mathrm{~nm}$ ) to focus a NIR laser at the precise imaging plane of a light microscope. We predicted that these micro ${ }^{540}$-UCPs would be effective due to their high efficiency and reported response to $\mathrm{NIR}^{28}$. As expected, micro ${ }^{540}$-UCPs can be excited with NIR and emit light in the visible range with a bright $\left(\sim 1100+\right.$ photoelectrons) emission intensity over background ${ }^{28}$. However, the micro ${ }^{540}$-UCPs also exhibit efficient inter-particle energy transfer and sublinear power dependence for emission (emission $=\operatorname{power}^{\mathrm{n}}$ where $\mathrm{n}<1$ ) at $>25,000$ Suns, which results in a beam broadening by $\sim 8 \mu \mathrm{m}$. The broadening is greater than the median particle size distribution full width $(\sim 2.2 \mu \mathrm{m})$. This results in suboptimal performance for micro ${ }^{540}$-UCPs in NIR beam coalignment, despite being demonstrably more efficient and having superior texture for focusing with transmitted visible light.

We next tested nano-UPCs ${ }^{29}$. Nano-UCPs can also be excited with NIR and also emit light in the visible range with a bright ( $\sim 500+$ photoelectrons $)$ emission intensity over background. However, in contrast to the micro ${ }^{540}$-UCPs, the smaller $27 \mathrm{~nm}$ diameter nanoUCPs showed a superlinear power dependence for emission (emission $=\operatorname{power}^{\mathrm{n}}$ where $\mathrm{n}>1$ ) which effectively reduced beam broadening $(\sim 2 \mu \mathrm{m})$ even at $>25,000$ Suns. The relative importance of emission intensity and particle size were not previously appreciated in selecting UCPs for microscope alignment. Furthermore, the nano-UCPs were superior to dual-NIR and visible camera alignment for efficient IR-LEGO (Infrared Laser-Evoked Gene Operator), an in vivo optogenetics assay using NIR to induce a heat-shock promoter driven expression of Yellow Fluorescent Protein (YFP) in the budding yeast Sacchromyces cerevisiae. Together, our results indicate that the lower efficiency nano-UCPs are better suited for coalignment of a NIR beam with the visible light path due to the retention of beam sharpness, attributable to their limited inter-particle energy transfer, small size, and lower efficiency.

\section{Results and Discussion}

Near-infrared light is both invisible to the human eye and spectrally separate from the visible wavelengths used in biological imaging by up to $1 \mu \mathrm{m}$ (Figure 1A), complicating coalignment at the imaging plane on a light microscope. To address this, the use of phosphor UCPs was investigated to directly translate the position of a NIR beam into visible light at the same focal plane. These UCPs are excited by NIR light and emit light at specific visible 
light wavelengths via an anti-Stokes mechanism ${ }^{21-25}$ (Figure 1A). We first investigated two types of micro-UCPs, which are predicted to respond to wavelengths in the NIR with emission in the visible range (micro ${ }^{540}$-UCPs and micro ${ }^{490}$-UCPs). Micro-UCPs were applied to glass coverslips with a random particle distribution to function as fiducial marks for precision focusing and mounted in water on a slide to mimic a typical microscopy set-up (Figure S1A). Imaging was performed on a spinning disc confocal microscope equipped with high-resolution optics commonly used for time lapse analysis of fluorescently tagged molecules ${ }^{30-31}$ and a fiber-coupled NIR multimode laser $(1470 \mathrm{~nm})$. Upon excitation with $1470 \mathrm{~nm}$ laser light, one of the two tested micro-UCPs, micro ${ }^{540}$-UCPs, emitted visible light (three-photon upconversion) detectable with a cooled CCD camera through a $525 \pm 25 \mathrm{~nm}$ band pass filter, and the emission intensity for these micro ${ }^{540}$-UCPs was bright at $\sim 1100$ photoelectrons above the background intensity (Figure S1B). The other micro-UCPs (micro 490 -UCPs; Figure S4A) did not respond to NIR excitation at any power with any visible light detectable though our emission filters $(525 \pm 25 \mathrm{~nm}$ or $620 \pm 25 \mathrm{~nm})$. This is consistent with the micro ${ }^{490}$-UCPs peak emission outside the transmission window of our emission filters. Importantly, the visible light camera used for optical imaging did not respond to NIR illumination in the absence of UCPs (Figure 2E). Thus, one of the two types of micro-UCPs respond to NIR excitation with an emission detectable on a standard highresolution microscope set up.

A power dependence study was performed for the micro ${ }^{540}$-UCPs that responded to NIR light. The fluorescence intensity at $525 \pm 25 \mathrm{~nm}$ was quantified following $1470 \mathrm{~nm}$ laser excitation with increasing laser power. Laser power density was measured using a power meter sensitive to $1470 \mathrm{~nm}$ light. Micro ${ }^{540}$-UCPs showed increased visible light emission responses to increased $1470 \mathrm{~nm}$ laser power from 3.2 to $181.3 \mathrm{~mW}$ with a power density of $\sim 4.8$ to $27.5 \times 10^{4}$ Suns $\left(\mathrm{kW} / \mathrm{m}^{2}\right)$ (Figure $\left.1 \mathrm{~B}, \mathrm{C}\right)$. The power dependence is plotted in a log$\log$ plot (log of emission intensity normalized to emission at the highest power versus $\log$ of power in $\mathrm{mW}$ ), since in these plots the slope will equal the order " $\mathrm{n}$ " of the power dependence for a given wavelength (emission $=$ laser power $^{\mathrm{n}}$ ). Because this upconversion is a three-photon process (1470 $\mathrm{nm}$ excitation and $525 \pm 25 \mathrm{~nm}$ emission), the observed decay in the power dependence from $\mathrm{P}^{0.88}$ to $\mathrm{P}^{0.85}$ with increasing $1470 \mathrm{~nm}$ laser power can be attributed to the saturation of the intermediate energy states ${ }^{32}$ (Figure 1B, C).

The size and composition of the micro ${ }^{540}$-UCPs were determined. Scanning Electron Microscopy (SEM) image analysis revealed the micro ${ }^{540}$-UCPs were $2.1 \pm 0.07 \mu \mathrm{m}$ in diameter (Figure 1D). Energy Dispersion Spectroscopy (EDS) analysis showed that the composition of micro ${ }^{540}$-UCPs is consistent with a rare-earth doped $\mathrm{Gd}_{2} \mathrm{O}_{2} \mathrm{~S}$ crystal (Figure $1 \mathrm{E})^{33-34}$.

While most UCPs are known to be resistant to photobleaching (or loss of emission light) upon excitation with low to moderate power densities (up to $\sim 10,000$ Suns) ${ }^{33,34}$, we next sought to test these micro ${ }^{540}$-UCPs for susceptibility to photo bleaching following excitation with $1470 \mathrm{~nm}$ NIR at the extremely high power densities employed by a high-resolution light microscope (Figure 2A, B). The change in emission luminescence levels in the visible range $(525 \pm 25 \mathrm{~nm})$ was quantified over time before, during, and after 45 minutes of continuous irradiation with $1470 \mathrm{~nm}$ laser light at high power density (27,500 Suns). The 
micro ${ }^{540}$-UCPs were highly photo-stable and even showed an increase in emission light intensity over time (Figure 2A, B). This increase in intensity over time may be due to local heating, as a large amount of NIR was used (e.g. ${ }^{35}$; see also IR-LEGO results below). When NIR laser illumination was switched on and off for alternating 2 min intervals, the emission intensity from micro ${ }^{540}$-UCPs at $525 \pm 25 \mathrm{~nm}$ was tightly correlated with the on and off states of the $1470 \mathrm{~nm}$ laser over time (Figure 2C, D). Therefore, these micro ${ }^{540}$-UCPs respond quickly to NIR irradiation and are highly resistant to photo bleaching, even after prolonged constant irradiation with a high-power density of NIR light.

In general, the efficiency of upconversion is a function of crystal composition and morphology ${ }^{36-37}$; as particle size decreases, the relative surface area to volume ratio increases, increasing the probability of non-radiative decay at surface defects ${ }^{37}$. It has been shown that for small $(<15 \mathrm{~nm})$ nano-UCPs, surface defect density induced-non-radiative decay is the prevalent mechanism of energy loss, and therefore upconversion luminescence is greatly decreased ${ }^{38}$. Examination of the emission profile along linescans of individual micro ${ }^{540}$-UCPs show enhanced emission near the particle edges, consistent with the presence of surface defects that induce both radiative and non-radiative decay (Figure S2BD). The data show that the excitation persists at least close to the edge of the micro ${ }^{540}$ UCPs. There is an additional spatial broadening process related to energy transfer in the near surface region: particle-to-particle energy transfer in which the excitation is transferred to neighboring particles where it can undergo either radiative (luminescent) or non-radiative decay; for radiative decay, this effectively broadens the spatial distribution of the particles.

To directly test the rate of particle-to-particle energy transfer, single particles within a chain as well as small chains of five individual micro ${ }^{540}$-UCPs were irradiated with a $1470 \mathrm{~nm}$ laser (Figure 3A). The degree of NIR excitation light spreading relative to UCP emission in $\mathrm{X}-\mathrm{Y}$ was quantified by monitoring the emission intensity of a single micro ${ }^{540}-\mathrm{UCP}$ in relationship to the edge of the laser beam. NIR beam spreading accounted for $14 \%$ of the signal (relative to direct irradiation of the particle with the NIR beam) within $1 \mu \mathrm{m}$ of the NIR beam edge to $\sim 2.5 \%$ of the signal about $5 \mu \mathrm{m}$ away from the beam (Figure $3 \mathrm{~B}$ ). When all 5 particles within a chain were irradiated, the central particles in the chain had similar or increased emission light intensity relative to the primary particle in the chain (Figure 3C, D). When only the primary $\left(1^{\circ}\right)$ particle in a chain was irradiated with NIR light, the secondary neighboring particle(s) also showed emission at $525 / 50 \mathrm{~nm}$ (average $64 \%$ of the emission level compared to the $1^{\circ}$ particle, Figure 3C, D). This particle-to-particle energy transfer could also be seen in the $3^{\circ}$ particle (average $32 \%$ relative to the $1^{\circ}$ particle), the $4^{\circ}$ particle (average $20 \%$ relative to the $1^{\circ}$ particle), and even the $5^{\circ}$ particle at an average distance of 6 $\mu \mathrm{m}$ from the edge of the beam (average $12 \%$ relative to the $1^{\circ}$ particle) within a linear chain of 5 particles (Figure 3C, D). Thus inter-particle energy transfer can persist through a chain of neighboring particles and produce emission several $\mu \mathrm{m}$ away from the light source; note that the energy transfer does not require the particles to be in contact, only in proximity (e.g. less than $10 \mathrm{~nm}^{39}$ ), a situation present in micro-UCP films.

The utility of micro ${ }^{540}$-UCPs for high resolution coalignment of visible and $1470 \mathrm{~nm}$ light was tested by employing a mask to delimit the region of NIR laser illumination while monitoring the emission profile $(29 \mu \mathrm{m}$ circle, Figure $4 \mathrm{~A})$. The micro ${ }^{540}$-UCPs allowed 
focusing of a NIR laser directly at the imaging plane and using the visible light-sensitive CCD camera. To quantify beam profiles, the following analysis was performed: (1) the laser excitation was first scaled by the measured micro ${ }^{540}$-UCPs power dependence, which was $\mathrm{P}^{0.85}$ at high power $(181.3 \mathrm{~mW})$; (2) the power adjusted laser profile was fit to a trapezoidal function to remove the noise (Figure 4B); and (3) the trapezoidal function was convoluted with a broadening function to simulate the "effective" particle size distribution (Figure 4B, D). The effective particle size distribution takes into account the transfer of excitation between particles (e.g. Figure 3); thus, for the purpose of fitting the emission profiles, the particle size distribution is broader than the physical particle size distribution (average 2.1 $\mu \mathrm{m})$. Of the probability distribution functions commonly used in spectroscopy, a Lorentzian function was chosen to represent the effective particle size distribution while the $\mathrm{R}^{2}$ values for the Lorentzian and Gaussian function are similar (0.993 with a Lorentzian PDF versus 0.992 with a Gaussian PDF), inspection of the fits show the Lorentzian is far more accurate in fitting the tails for the distribution consistent with a very broad effective particle size distribution due to high particle-to-particle energy transfer. (Note that similar $\mathrm{R}^{2}$ values result from the $\mathrm{R}^{2}$ calculations being mostly heavily weighted by the fit at the center of the spatial profile since these are the points of maximum amplitude). This analysis showed that the micro-UCP emission profile tails were best fitted with a Lorentzian resolution function of Full Width Half Max (FWHM) $=7.78 \mu \mathrm{m}$ (Figure 4C, D). Thus, although micro ${ }^{540}$-UCPs can be used to co-align a NIR laser beam with the visible imaging plane of a light microscope, the large size and efficient particle-to-particle luminescence transfer leads to substantial beam broadening.

To improve on this beam broadening, testing was performed with two smaller and less efficient nano-UCPs for NIR and visible coalignment, nano ${ }^{545}$-UCPs and nano ${ }^{538}$-UCPs $^{29}$. Both nano-UCPs are not predicted to respond to wavelengths longer than $1000 \mathrm{~nm}$ with emission in the visible range $(545 \mathrm{~nm} \text { and } 538 \mathrm{~nm} \text {, respectively })^{40-41}$; however, it was hypothesized that the high-power densities of NIR on a high-resolution microscope $(>25,000$ Suns) may trigger an emission response from $1470 \mathrm{~nm}$ light that is not observed under low power densities. The nano-UCPs were applied to glass coverslips and imaged on a spinning disc confocal microscope with a CCD camera as was done for micro-UCPs (Figure S1A). Upon excitation with $1470 \mathrm{~nm}$ laser light, both nano-UCPs emitted visible light (threephoton upconversion) detectable through a $525 \pm 25 \mathrm{~nm}$ band pass filter (Figure S3A). For the nano ${ }^{545}$-UCPs, the emission intensity was sufficiently bright at $\sim 500$ photoelectrons above the background intensity (Figure S3A). For the nano ${ }^{538}$-UCPs, the emission response was less efficient, although still readily detectable with longer $(2 \times)$ exposure times $(\sim 300$ photoelectrons above the background intensity; Figure S4B-F). Therefore, nano-UCPs, like micro ${ }^{540}$-UCPs, emit visible light in response to excitation with both visible light and NIR $(1470 \mathrm{~nm})$ light (Figure S3A-D).

Next, a power dependence study was performed for the nano-UCPs. Like the high-efficiency micro ${ }^{540}$-UCPs, both nano-UCPs showed increased visible light emission responses to increased $1470 \mathrm{~nm}$ laser power from 3.2 to $181.3 \mathrm{~mW}$ with a power density of $\sim 4.8$ to $27.5 \times$ $10^{4}$ Suns (nano ${ }^{545}$-UCPs Figure 5A, B) and from 137 to $181.3 \mathrm{~mW}$ with a power density of $\sim 20.75$ to $27.5 \times 10^{4}$ Suns (nano ${ }^{538}$-UCPs Figure S4B). The power dependence is again plotted in a log of emission intensity (normalized to emission at the highest power) versus 
$\log$ of power (mW) since in these plots the slope will equal the order " $n$ " of the power dependence for a given wavelength (emission $=$ laser power $^{\mathrm{n}}$ ). Because this upconversion is a three-photon process (1470 nm excitation and $525 \pm 25 \mathrm{~nm}$ emission), the observed power dependencies decay from $\mathrm{P}^{2.17}$ to $\mathrm{P}^{1.92}$ for the nano ${ }^{545}$-UCPs (and from $\sim \mathrm{P}^{3.00}$ to $\mathrm{P}^{2.67}$ for the nano ${ }^{538}$-UCPs) with increasing $1470 \mathrm{~nm}$ laser power (Figure 5A, B) can be attributed to the partial saturation of the intermediate energy states, albeit with a lower efficiency than observed with micro ${ }^{540}$-UCPs (Figure 1B, C) ${ }^{32}$. Since the power dependence is higher for the nano ${ }^{538}$-UCPs (Figure S4B) than the nano ${ }^{545}$-UCPs (Figure 5A, B), the data is consistent with shorter lifetimes of the intermediate excited states of nano ${ }^{538}$-UCPs being responsible for their lower efficiency, and the low overall visible light emission of the nano ${ }^{538}$-UCPs is consistent with a smaller pre-exponential constant for efficiency of emission at $525 \pm 25 \mathrm{~nm}$.

The size distribution and chemical composition of the nano ${ }^{545}$-UCPs were determined. Atomic Force Microscopy (AFM) image analysis revealed that the nano ${ }^{545}$-UCPs were on average $27.22 \pm 0.81 \mathrm{~nm}$ (standard error) in diameter (Figure 5G). The height distribution analysis was complicated due to the PEG supplied with the nano ${ }^{545}$-UCPs, which forms a thick film surrounding the nano-UCPs during sample preparation for AFM (Figure 5I), leading to a lower measured average height $(5.59 \pm 0.13 \mathrm{~nm}$ standard error; e.g. Figure $5 \mathrm{H})$. Energy Dispersion Spectroscopy (EDS) analysis showed that the composition of nano ${ }^{545}$ $\mathrm{UCPs}$ is consistent with a rare-earth doped $\mathrm{NaYF}_{4}$ crystal (Figure 5J). It is noted that the PEG may also help space the nano-UCPs apart in the thin film, thereby decreasing particle to particle energy transfer.

To test for susceptibility to photo bleaching following excitation with NIR at $27.5 \times 10^{4}$ Suns $\left(\mathrm{kW} / \mathrm{m}^{2}\right)$ on a light microscope (Figure $5 \mathrm{C}, \mathrm{E}$ ), the change in emission luminescence levels in the visible range $(525 \pm 25 \mathrm{~nm})$ was quantified for nano ${ }^{545}$-UCPs over time before, during, and after 45 minutes of continuous irradiation with $1470 \mathrm{~nm}$ laser light. Like the micro ${ }^{540}$-UCPs, nano ${ }^{545}$-UCPs were highly photo-stable but did not exhibit any increase (or decrease) in emission intensity after 45 minutes of continuous NIR irradiation (Figure 5C, E). Furthermore, when NIR laser illumination was switched on and off for alternating 2 min intervals, the emission intensity from nano ${ }^{545}$-UCPs at $525 \pm 25 \mathrm{~nm}$ was tightly correlated with the on and off states of the $1470 \mathrm{~nm}$ laser over time (Figure 5D, F). Importantly, no emission signal was detected with the visible light camera in the absence of UCPs when the NIR laser is on (Figure 2E). Together these data suggest that nano ${ }^{545}$-UCPs, like the micro ${ }^{540}$-UCPs, are highly photo-stable even with very high power density excitation light (>25,000 Suns),

The relative utility of the nano-UCPs for high resolution coalignment of visible and $1470 \mathrm{~nm}$ light was quantified using a mask to delimit the region of NIR laser illumination while monitoring the emission profile as was done above for micro ${ }^{540}$-UCPs ( $29 \mu \mathrm{m}$ circle, Figure 6A). Nano-UCPs have a small median diameter (Figure $5 \mathrm{G}-\mathrm{I}$ ) ${ }^{40-41}$, but the effective particle size distribution takes into account that the excitation can be transferred between particles. Therefore, for fitting the emission profiles, the particle size distribution is broader than the physical particle size distribution (Figure 6B, C). A Lorentzian function for the nano ${ }^{545}$ UCPs again demonstrated a marginally higher $\mathrm{R}^{2}$ value as compared to a Gaussian 
probability distribution function (PDF) (0.993 with a Lorentzian PDF versus 0.992 with a Gaussian PDF), with a FWHM of $2.33 \mu \mathrm{m}$ (Figure 6D). A Lorentzian PDF showed a similar $\mathrm{R}^{2}$ to a Gaussian distribution for the nano ${ }^{538}$-UCPs $\left(\mathrm{R}^{2}\right.$ value $=0.9923$ vs 0.9925 with a Gaussian PDF) with a FWHM of $1.66 \mu$ m using a Lorentzian distribution (Figure S4F). The experimental emission profiles for both nano-UCPs had narrower FWHM than the excitation laser's profile taken with the NIR sensitive camera (Figure 6A, B), likely because the reduced efficiency (Figure 5A, B; S4B) results in reduced particle-to-particle energy transfer.

Finally, the nano ${ }^{545}$-UCPs were compared with a NIR-sensitive camera for NIR and visible light coalignment at the specimen plane (Figure S5) in an optogenetics assay. An InfraredLaser-Evoked Gene Operator (IR-LEGO) ${ }^{2}$ assay was developed in budding yeast $S$. cerevisiae with a genetically encoded $4 \times$ repeated heat shock element $(4 \times h s e)$ promotordriven Yellow Fluorescent Protein (YFP) expression construct (Figure 7A, B) ${ }^{42-43}$. This hse promoter is activated upon shifting the growing yeast from normal growth temperature (25$\left.30^{\circ} \mathrm{C}\right)$ to a higher more stressful temperature $\left(35-43^{\circ} \mathrm{C}\right)$ by the heat-sensitive transcription factor Heat Shock Factor 1 (Hsf1) (Figure 7A, for review see $\left.{ }^{44}\right)^{42}$. A 1470 nm NIR laser was used to induce local increase in temperature due to a peak in absorption for water (the major component of cellular cytoplasm) at that wavelength ${ }^{2}$. The NIR laser was first aligned with visible light at the imaging plane using either the one step nano ${ }^{545}$-UCPs or an NIRsensitive camera for coalignment. The yeast were grown at low temperature $\left(25^{\circ} \mathrm{C}\right)$ and then mounted in a thermally controllable imaging chamber ${ }^{31}$ set to $25^{\circ} \mathrm{C}$. An image prior to IRLEGO was acquired to determine the background levels of YFP intensity per pixel per yeast (Figure 7B, C). A $29 \mu \mathrm{m}$ circle mask was used to delimit the region of $1470 \mathrm{~nm}$ NIR laser irradiation $(10.8 \mathrm{~mW})$ on a subset of yeast within the field of view (Figure 7C). The NIR laser was maintained on the yeast for 2 hours with simultaneous time-lapse imaging of YFP fluorescence every 12 minutes throughout NIR irradiation (Figure 7B). The IR-LEGO efficiency was calculated as a ratio of the change in YFP signal for yeast inside the NIRirradiated region relative to the signal for yeast outside of the NIR-irradiated region (Figure 7C). Alignment of the microscope using nano ${ }^{545}$-UCPs led to a higher average IR-LEGO efficiency than alignment with the NIR camera, with an average $11.7 \%$ increase in peak IRLEGO-induced YFP levels using nano ${ }^{545}$-UCPs for NIR laser alignment versus an average peak increase of $4.4 \%$ using the patterned metal slide and NIR-sensitive camera $(\mathrm{p}=0.0161$ using students t-test) (Figure 7D, E). Together, these data demonstrate that nano-UCPs are an improved tool for high-resolution alignment of a NIR laser on a light microscope for in vivo biological experiments.

\section{Conclusions}

UCPs allow a one-step, one camera method (Figure S5) to co-align an NIR laser with the specimen plane on a light microscope. Both micro ${ }^{540}$-UCPs and nano ${ }^{545}$-UCPs are (a) highly photo-stable and respond quickly to the onset and cessation of NIR excitation with emitted light, (b) show an emission peak in the green visible range with two- and threephoton excitation, and (c) respond to increased power of excitation light with increased levels of emission light even at very high power density ( $>25,000$ Suns). While it might be expected that micro ${ }^{540}$-UCPs would be ideal for coalignment of NIR and visible optical 
paths, their high efficiency makes them less suitable. The increased efficiency of the larger micro ${ }^{540}$-UCPs, relative to the smaller nano-UCPs, is consistent with longer-lived excited states for larger particles due a low surface area to volume ratio ${ }^{38}$. The long-lived excited state enables particle-to-particle energy transfer and broadens the particle size distribution from $2.1 \mu \mathrm{m}$ (mean diameter) to $7.8 \mu \mathrm{m}$ (FWHM Lorentzian) for the micro ${ }^{540}$-UCPs. In contrast, nano-UCPs were clearly superior for precise alignment due to their smaller size and higher power dependence. The higher power dependence and short-lived excited states ${ }^{38}$ of the nano-UCPs facilitate more precise focusing of the NIR laser because they induce a sharpening of the spatial distribution of the emission profile relative to the excitation profile. Precision in NIR laser detection and alignment is important for high-resolution microscopy of biological samples for optogenetics analysis, which typically examines micrometer- (or even sub-micrometer-) sized particles and structures within specific cells or tissues. For an IR-LEGO in vivo optogenetics assay in yeast, pre-experimental alignment of the NIR laser with the visible light imaging plane of the microscope with the nano-UCPs produced a more robust IR-LEGO response than traditional dual camera methods (Figure S5). In vivo optogenetics assays require extreme precision; thus, these luminescent nano-UCPs will be powerful tools to increase experimental accuracy and reproducibility in combinatory NIR and visible light microscopy. Furthermore, due to their small size (point sources of light), these nano-UCPs could also be used to measure the point-spread functions and calculate the chromatic shift throughout the field of view. It is expected that phosphor UCPs have the potential for wide use as tools in modern light microscopy.

\section{Methods}

\section{Upconverting nanoparticles and preparation of coated slides for microscopy}

PTIR545/UF (micro ${ }^{540-U C P s) ~ w e r e ~ a ~ g i f t ~ f r o m ~ P h o s p h o r ~ T e c h n o l o g y, ~ L t d . ~(U K) . ~ S u n s t o n e ~}$ nano ${ }^{545}$-UCPs and nano ${ }^{538}$-UCPs were obtained from Sigma Aldrich (\#90992 and \#75207 respectively). ISPRG particles (Micro ${ }^{490}$-UCPs) were obtained from MaxMax.com (Figure S4A). Micro-UCPs were suspended in 100\% ethanol and vortexed vigorously (Figure S1A). A drop of this suspension was deposited on a $22 \times 22 \mathrm{~mm}$ No. 1.5 coverslip and allowed to dry (Figure S1A). The coverslip was placed on top of a standard microscopy slide, sandwiched with a small drop of distilled water and sealed with clear nail polish around the edges (Figure S1A). Nano ${ }^{545}$-UCPs and nano ${ }^{538}$-UCPs were suspended in distilled water as per the manufacturer's instructions. About $5-10 \mu \mathrm{L}$ of this water-UCP solution was pipetted onto a $22 \times 22 \mathrm{~mm}$ No. 1.5 coverslip and allowed to dry. The coverslip was then placed on top of a standard microscopy slide, sandwiched with a small drop of distilled water and sealed with clear nail polish (mostly nitrocellulose dissolved in ethyl acetate and butyl acetate) around the edges (Figure S1A).

\section{Laser power measurement}

The power of the $1470 \mathrm{~nm}$ NIR laser was measured using XLP12-3S-H2-DO scan head and an accompanying Maestro power monitor (Gentec-EO) by varying the laser power with Syscon Geo software (Rapp Optoelectonics $\mathrm{GmbH}$ ) and measuring the corresponding increase in $\mathrm{mW}$. 


\section{Spinning disc confocal imaging system}

Upconverting nanoparticles were imaged on a spinning disc confocal unit (CSU-10; Yokogawa) upgraded with Borealis (Spectral Applied Research) on an inverted Ti microscope (Nikon) with a $60 \times 1.4$ NA oil-immersion Plan Apo objective. For images in the experiments to test power dependence of micro ${ }^{540}$-UCPs (Figures 1B, 1C), curve fitting of emission profiles of micro ${ }^{540}$-UCPs, nano ${ }^{545}$-UCPs and nano ${ }^{538}$-UCPs (Figures 4, 6 and S4 respectively), power dependence and photostability of nano ${ }^{545}$-UCPs (Figure 5A-F) and power dependence of nano ${ }^{538}$-UCPs (Figure S4B), emission of nano ${ }^{545}$-UCPs in the visible region upon excitation with NIR or visible light (Figures S3A and S3D respectively), to test the reaction of micro ${ }^{540}$-UCPs with high temporal resolution (Figure S2A) and to test the emission in the 525/50 channel upon $1470 \mathrm{~nm}$ irradiation with no sample present (Figure $2 \mathrm{E}), 2 \times 2$ binning and a gain of 1 was used. For images to test the photostability of micro ${ }^{540}$-UCPs (Figure 2A-D), particle to particle transfer (Figure 3), emission of micro ${ }^{540}$ UCPs in the visible region upon excitation with NIR or visible light (Figure S1B, S1E), emission profile of a single micro ${ }^{540}$-UCP (Figure S2B-D), no binning and a gain of 1 was used. Long term imaging of micro ${ }^{540}$-UCPs in Figure 2 was performed in a temperaturecontrolled device set to $21^{\circ} \mathrm{C}$ (Bioptechs) $)^{31}$. Laser excitation was either provided by a $\sim 3 \mathrm{~W}$ $1470 \mathrm{~nm}$ laser (Rapp Optoelectronics $\mathrm{GmbH}$ ) or by using an acousto-optic tunable filter to select the excitation light from either of two $100 \mathrm{~mW}$ lasers for excitation at 491 and $561 \mathrm{~nm}$ respectively (Spectral Applied Research). A filter wheel was used for emission wavelength selection (Sutter Instrument) with 2 bandpass filters corresponding to 525/50 nm and 620/50 $\mathrm{nm}$ for collecting emission light. A cooled charge-coupled device (CCD) camera (Orca-R2; Hamamatsu Photonics) was used for image acquisition in the visible range. An IR sensitive camera (Goldeye P-008, Allied Vision) was used for capturing NIR light (Figure 4A and 6A). During NIR irradiation experiments, focus was maintained relative to the coverslip using CRISP auto focus system (Applied Scientific Instrumentation) before each acquisition. The system was controlled with MetaMorph software (Molecular Devices).

\section{Multiphoton imaging system}

Spectral multiphoton imaging (Figures S1C, D and S3B, C) was performed with an A1RMP laser scanning system on an Eclipse Ti-E microscope stand using a 25× 1.1 NA ApoLWD water- immersion objective (Nikon). The A1 spectral detector was set to collect emission from 400 to $650 \mathrm{~nm}$ in $10 \mathrm{~nm}$ increments. NIR illumination was provided by a Chameleon Vision II tunable laser (Coherent, Santa Clara, CA) without a pinhole. For NIR illumination scanning, images were collected with the laser tuned as close as possible to the absorption maximum of the nanoparticles (micro ${ }^{540}$ UCPs: $_{970} \mathrm{~nm}$ and nano ${ }^{545}$-UCPs: $980 \mathrm{~nm}$ ), and then at 50-nm intervals from $700 \mathrm{~nm}$ to $1000 \mathrm{~nm}$, using the same detector gain, pixel size, and dwell time. Relative laser power was kept constant at the sample by adjusting the acousto-optic modulator (AOM) as the wavelength was tuned.

\section{Atomic force microscopy}

To elucidate the size distribution of individual nano ${ }^{545}$-UCPs (Figure 5G-I), tapping mode atomic force microscopy (AFM) was employed using Si tips (Bruker). A diluted solution (4 $\mathrm{mg} / \mathrm{mL}$ ) containing nanoparticles was dropped on p-doped Si substrates; afterwards, the wet 
samples were dried in a vacuum oven at $70^{\circ} \mathrm{C}$ for $1 \mathrm{hr}$. All AFM measurements were carried out under ambient conditions.

\section{Scanning Electron Microscopy (SEM) \& Electron Dispersion Spectroscopy (EDS)}

Scanning Electron Microscopy (SEM) with concurrent Electron Dispersion Spectroscopy (EDS) was performed in order to analyze the size distribution and elemental composition of the particles. SEM images were taken for micro ${ }^{540}$-UCPs (Figure 1D) using a Zeiss Sigma $500 \mathrm{FE}$-SEM. SEM was performed on Si substrate at an accelerating voltage of $10 \mathrm{KeV}$. EDS for the micro ${ }^{540}$-UCPs (Figure 1E) and nano ${ }^{545}$-UCPs (Figure 5J) was performed on both $\mathrm{Si}$ and carbon substrates for both particle types using an FEI XL30 E-SEM equipped with an Oxford EDS detector, and then cross-referenced across substrates for accuracy. All samples were drop cast onto their respective substrates by first suspending in water/ethanol mixture $(\sim 1 \mathrm{mg} / \mathrm{mL})$ via sonication and stirring, then depositing $1 \mu \mathrm{L}$ on the substrate. The wet samples were dried in a vacuum oven at $70^{\circ} \mathrm{C}$ for $1 \mathrm{hr}$.

\section{Optical Microscopy Image acquisition and analysis}

Image analysis (including intensity measurements and line scan analysis) were performed using Fiji (ImageJ) software and graphs were generated in Prism 6 software (Graphpad software, Inc.). To convert intensity values from grayscale arbitrary units to the number of photoelectrons, a photon transfer curve was plotted ${ }^{45}$ to determine a conversion factor $\mathrm{K}$ ( $\mathrm{K}=4.32$ for no binning imaging condition and $\mathrm{K}=8.28$ for $2 \times 2$ binning imaging condition) that was then used to perform the conversion to number of photoelectrons per grayscale intensity value. For images in experiments to test emission of micro ${ }^{540}$-UCPs and nano ${ }^{545}$ UCPs in the visible region upon excitation with NIR or visible light (Figure S1B, S1E and S3A, S3D respectively), photostability of micro ${ }^{540}$-UCPs and nano ${ }^{545}$-UCPs (Figures $2 \mathrm{~B}$, $2 \mathrm{D}$ and $5 \mathrm{E}, 5 \mathrm{~F}$ respectively). mean intensity values were measured with a Region of Interest (ROI) of $\sim 16 \mu \mathrm{m}^{2}$ from a single Z plane image. For Figure S1B and S3A, similar sized ROIs were placed away from the region of irradiation to generate the background intensity. For Figure S1E and S3D intensity measurement of the no excitation image was used for background subtraction. For the images acquired in the multiphoton spectral imaging (Figures S1C, S1D, S3B and S3C), mean intensity values were measured from a single Z plane image for each wavelength across the spectrum. For the kymographs in Figure 2A, C and 5C, D, a 5-pixel thick line was drawn along the image and the Image re-slice function in Fiji was used to generate the kymograph. For quantification of images in experiments to test power dependence of micro ${ }^{540}$-UCPs, nano ${ }^{545}$-UCPs, micro ${ }^{490}$-UCPs and nano ${ }^{538}$-UCPs (Figures 1B \& 1C, 5A \& 5B, S4A and S4B respectively), photostability of micro ${ }^{540}$-UCPs and nano ${ }^{545}$-UCPs (Figures $2 \mathrm{~B}, 2 \mathrm{D}$ and $5 \mathrm{E}, 5 \mathrm{~F}$ respectively) and to test the reaction of micro ${ }^{540}$-UCPs with high temporal resolution (Figure S2A), a square ROI of $\sim 16 \mu \mathrm{m}^{2}$ was used to measure mean intensity. 2 similar ROIs in the background were used to generate the background mean intensity. For the linescans to quantify the emission profile of single micro ${ }^{540}$-UCPs (Figure S2B-D), a 3-pixel thick line was drawn along the middle plane of the Z-sections and the plot profile function in Fiji was used to generate the linescan. A similar line was drawn away from the region of irradiation to generate the background linescan which was then subtracted. 


\section{Power Dependence Analysis}

micro ${ }^{540}$-UCPs, nano ${ }^{545}$-UCPs, micro ${ }^{490}$-UCPs and nano ${ }^{538}$-UCPs were excited with a $1470 \mathrm{~nm}$ laser and the power was varied from $3.18 \mathrm{~mW}$ to $181.33 \mathrm{~mW}$. For micro ${ }^{490}$-UCPs, the emission intensity was recorded for each step up in laser power, and the data points were plotted in a linear graph (Figure S4A) while for micro ${ }^{540}$-UCPs, nano ${ }^{545_{-} \text {UCPs and nano }}{ }^{538}$ UCPs, the emission intensity was recorded for each step up in laser power and the data points were plotted in log-log plot (Figure 1B, 1C, 5A, 5B and S4B). For micro ${ }^{540}$-UCPs and nano ${ }^{545}$-UCP, power dependence was compared at low power vs. high power to study the saturation effect on each particle type. It is noted that significant noise is associated with the power meter measurements of the excitation power at low laser powers.

\section{Particle to Particle Energy Transfer}

To test particle to particle energy transfer (Figure 3B, 3C and 3D), 15 or $16 \mathrm{Z}$-sections spaced $200 \mathrm{~nm}$ apart were acquired and a sum- projected image was generated from the Zsections of the 525/50 channel. ROIs of the micro ${ }^{540}$-UCPs either single particle or in chains were drawn by tracing the outline of the particles in the $525 / 50 \mathrm{~nm}$ sum projected image to measure its mean intensity (Figure 3C). Two ROIs of $\sim 16 \mu \mathrm{m}^{2}$ were used to generate the background mean intensity from the sum projected image which was then subtracted from the measurement.

\section{Beam profile fit analysis}

For the beam profile fitting of micro ${ }^{540}$-UCPs, nano ${ }^{545}$-UCPs and nano ${ }^{538}$-UCPs (Figure 4A, 4C, 6A, 6C, S4C and S4E respectively), a 5-pixel thick line was drawn along the image and the plot profile function in Fiji was used to generate the linescan. The line was then rotated $45^{\circ}$ and another linescan was performed. This was repeated twice more to generate a total of 4 linescans per image which was then averaged. A similar analysis was performed away from the region of irradiation to generate the average background linescan which was subtracted from the measurement.

Fitting was performed using MATLAB's built-in genetic learning algorithm. Further optimization and validation of the results from the genetic algorithm was performed using a non-linear least squares minimization. First, the laser profile was adjusted using the power dependence of emission at $100 \%$ laser power for micro ${ }^{540}$-UCPs (Figure 4B), nano ${ }^{545}$-UCPs (Figure 6B) and nano ${ }^{538}$-UCPs (Figure S4D) and then the recorded emission profiles (Figures 4D, 6D and S4F for micro ${ }^{540}$-UCPs, nano ${ }^{545_{-U C P s} \text { and nano }}{ }^{538}$-UCPs respectively) and adjusted response profiles (Figure 4B, 6B and S4D in magenta) were normalized to 1 . Subsequently, the laser profiles were fitted to trapezoids representing an ideal power scaled response for each particle, using the $\mathrm{R}^{2}$ value of the fit only on the steep slopes as a fitness metric, to avoid fitting the noise at the peak emission and baseline. These ideal response profiles are shown in cyan in Figure 4B, 6B and S4D for micro ${ }^{540}$-UCPs, nano ${ }^{545}$-UCPs and nano ${ }^{538}$-UCPs respectively. This trapezoidal profile was convoluted with a Lorentzian distribution function, which was fitted to the emission profiles of each UCP (dashed line in Figures 4D, 6D and S4F for micro ${ }^{540}$-UCPs, nano ${ }^{545}$-UCPs and nano ${ }^{538}$ UCPs respectively). The full width half max (FWHM) of the Lorentzian probability distribution function (shown in yellow in Figures 4D, 6D and S4F for micro ${ }^{540}$-UCPs, 
nano ${ }^{545}$-UCPs and nano ${ }^{538}$-UCPs respectively) was varied to obtain the best $\mathrm{R}^{2}$ value over the totality of the function domain, which naturally weights the tails of the emission profiles more than the slopes at the sides, since the tails contain significantly more data points. The solution to the mixed-integer multifunction optimization was transferred to a non-linear least squares minimization algorithm as the starting points, thus validating the local minimum to the highest accuracy possible. The upper and lower bounds as well as all other parameters used for the genetic algorithm and non-linear least squares were identical for both particles.

\section{IR-LEGO experiment}

S. cerevisiae strain DPY144 was a kind gift from Dr. David Pincus (Whitehead Institute, MIT $)^{42}$. Yeast were grown on YPD plates ${ }^{46}$ and cultivated at $25^{\circ} \mathrm{C}$. For imaging, single colonies were cultivated at $25^{\circ} \mathrm{C}$ (with shaking) in synthetic complete (SC) media ${ }^{46}$ for 10 $12 \mathrm{hr}$, then diluted to early-log phase $\left(\mathrm{OD}_{600}=0.1\right)$ and grown for $3 \mathrm{hr}$ (to mid-log phase) prior to imaging. Yeast cells were mounted in a fluidic temperature control device ${ }^{31}$ to stably maintain sample temperature at $25^{\circ} \mathrm{C} .10 \mu \mathrm{L}$ of yeast cell suspension was loaded onto a $2 \%$ agarose pad within the specimen chamber. Images were acquired on the spinning disc confocal microscope described above with a $60 \times 1.4$ NA oil-immersion Plan Apo objective with $2 \times 2$ binning. For transmitted light, a single $Z$-plane in the center of the yeast was acquired every $3 \mathrm{~min}$. For YFP imaging, an $8 \times 1 \mu \mathrm{m} Z$-series through the yeast was collected every $12 \mathrm{~min}$ for $\sim 2.5$ hour using the $488 \mathrm{~nm}$ laser for excitation with the 525/50 emission filter. Following the initial time point, a circular region ( $29 \mu \mathrm{m}$ diameter) was targeted with $10.8 \mathrm{~mW} 1470 \mathrm{~nm}_{\text {laser }}^{2}$ to gently and locally heat the sample within that region for $2 \mathrm{hr}$, after which the NIR irradiation was turned off and the sample imaged for another $\sim 30 \mathrm{~min}$. Peak YFP emission intensity was reached at $\sim 96 \mathrm{~min}$ after the onset of IRLEGO.

\section{IR-LEGO data analysis}

The YFP images were maximum projected for analysis in FIJI (Image J). Yeast cells were segmented from the image by thresholding in which the images were binarized, and subjected to watershed analysis to identify discrete yeast cells. The average pixel intensity of YFP signal within individual yeast cells both inside and outside the region of IR-LEGO was quantified. IR-LEGO efficiency was calculated as the ratio of pixel intensity inside the 29 $\mu \mathrm{m}$ NIR-targeted region to outside the IR-LEGO region at each time point. IR-LEGO efficiency at $\mathrm{t}=0$ and $\mathrm{t}=96 \mathrm{~min}$ after NIR irradiation initiation was compared by a parametric unpaired Student's t-test. Significance was set at $a=0.05$.

\section{Supplementary Material}

Refer to Web version on PubMed Central for supplementary material.

\section{Acknowledgments}

We thank all members of the Canman, Shirasu-Hiza, Dumont, and Kummel labs for advice and support. We thank Phosphor Technology, Ltd (UK) for kindly providing us with PTIR545/UF upconverting microparticles (micro 540 . UCPs). We thank Dr. Jennifer Waters (Nikon Imaging Center at Harvard Medical School); Chris Anderson (Biovision, Inc.); and Dr. Sven Wildfang (Rapp Optoelectronics GmBH) for technical microscope assistance. We thank Dr. Talley Lambert (Nikon Imaging Center at Harvard Medical School) for advice on photoelectron 
conversion. We thank Dr. Luke Berchowitz (Columbia University) for helpful discussions and assistance with yeast husbandry, and Dr. David Pincus (Whitehead Institute, MIT) for the DPY144 yeast strain. We thank Dr. Liza Pon (Columbia University) for use of yeast equipment, and members of the Pon lab (especially Dr. Istvan Boldogh) for helpful discussions and advice. Spectral multiphoton imaging was performed with help from Dr. Theresa Swayne in the Confocal and Specialized Microscopy Shared Resource of the Herbert Irving Comprehensive Cancer Center at Columbia University Medical Center. We thank Dr. William Trogler for critical comments on this manuscript. This work was funded by: 2P30CA023100-28S (AGB); a postdoctoral fellowship from the Charles H. Revson Foundation (TD); ANR-09-RPDOC-005-01 (JD); FRM-AJE201112 (JD); the Emergence program from Mairie de Paris (JD); NIH-R01-GM105775 (MSH); NIH-R01-AG045842 (MSH); NIH-U54CA132379 (ACK); NIHR01GM117407 (JCC); and NIH-DP2-OD008773 (JCC).

\section{References}

1. Deguchi T, Itoh M, Urawa H, Matsumoto T, Nakayama S, Kawasaki T, Kitano T, Oda S, Mitani H, Takahashi T, Todo T, Sato J, Okada K, Hatta K, Yuba S, Kamei Y. Infrared Laser-Mediated Local Gene Induction in Medaka, Zebrafish and Arabidopsis Thaliana. Dev Growth Differ. 2009; 51(9): 769-75. [PubMed: 19843153]

2. Kamei Y, Suzuki M, Watanabe K, Fujimori K, Kawasaki T, Deguchi T, Yoneda Y, Todo T, Takagi S, Funatsu T, Yuba S. Infrared Laser-Mediated Gene Induction in Targeted Single Cells in Vivo. Nat Methods. 2009; 6(1):79-81. [PubMed: 19079252]

3. Fazal FM, Block SM. Optical Tweezers Study Life under Tension. Nat Photonics. 2011; 5:318-321. [PubMed: 22145010]

4. Hoover EE, Squier JA. Advances in Multiphoton Microscopy Technology. Nat Photonics. 2013; 7(2):93-101. [PubMed: 24307915]

5. Supatto W, Truong TV, Debarre D, Beaurepaire E. Advances in Multiphoton Microscopy for Imaging Embryos. Curr Opin Genet Dev. 2011; 21(5):538-48. [PubMed: 21917444]

6. Mojzisova H, Vermot J. When Multiphoton Microscopy Sees near Infrared. Curr Opin Genet Dev. 2011; 21(5):549-57. [PubMed: 21924603]

7. Radosevich AJ, Bouchard MB, Burgess SA, Chen BR, Hillman EM. Hyperspectral in Vivo TwoPhoton Microscopy of Intrinsic Contrast. Opt Lett. 2008; 33(18):2164-6. [PubMed: 18794965]

8. Franck AD, Powers AF, Gestaut DR, Davis TN, Asbury CL. Direct Physical Study of KinetochoreMicrotubule Interactions by Reconstitution and Interrogation with an Optical Force Clamp. Methods. 2010; 51(2):242-50. [PubMed: 20096784]

9. Lang MJ, Fordyce PM, Engh AM, Neuman KC, Block SM. Simultaneous, Coincident Optical Trapping and Single-Molecule Fluorescence. Nat Methods. 2004; 1(2):133-9. [PubMed: 15782176]

10. Bansal A, Liu H, Jayakumar MK, Andersson-Engels S, Zhang Y. Quasi-Continuous Wave nearInfrared Excitation of Upconversion Nanoparticles for Optogenetic Manipulation of C. Elegans. Small. 2016; 12(13):1732-43. [PubMed: 26849846]

11. Gradinaru V, Zhang F, Ramakrishnan C, Mattis J, Prakash R, Diester I, Goshen I, Thompson KR, Deisseroth K. Molecular and Cellular Approaches for Diversifying and Extending Optogenetics. Cell. 2010; 141(1):154-65. [PubMed: 20303157]

12. He L, Zhang Y, Ma G, Tan P, Li Z, Zang S, Wu X, Jing J, Fang S, Zhou L, Wang Y, Huang Y, Hogan PG, Han G, Zhou Y. Near-Infrared Photoactivatable Control of $\mathrm{Ca}(2+)$ Signaling and Optogenetic Immunomodulation. Elife. 2015; 4:e10024. [PubMed: 26646180]

13. Bath DE, Stowers JR, Hormann D, Poehlmann A, Dickson BJ, Straw AD. Flymad: Rapid Thermogenetic Control of Neuronal Activity in Freely Walking Drosophila. Nat Methods. 2014; 11(7):756-62. [PubMed: 24859752]

14. Hososhima S, Yuasa H, Ishizuka T, Hoque MR, Yamashita T, Yamanaka A, Sugano E, Tomita H, Yawo H. Near-Infrared (Nir) up-Conversion Optogenetics. Sci Rep. 2015; 5:16533. [PubMed: 26552717]

15. Kaberniuk AA, Shemetov AA, Verkhusha VV. A Bacterial Phytochrome-Based Optogenetic System Controllable with near-Infrared Light. Nat Methods. 2016; 13(7):591-7. [PubMed: 27159085]

16. Richter CP, Rajguru S, Bendett M. Infrared Neural Stimulation in the Cochlea. Proc SPIE Int Soc Opt Eng. 2013; 8565:85651Y. 
17. Richter CP, Tan X. Photons and Neurons. Hear Res. 2014; 311:72-88. [PubMed: 24709273]

18. Neuman KC, Block SM. Optical Trapping. Rev Sci Instrum. 2004; 75(9):2787-809. [PubMed: 16878180]

19. Kim WJ, Nyk M, Prasad PN. Color-Coded Multilayer Photopatterned Microstructures Using Lanthanide (Iii) Ion Co-Doped Nayf4 Nanoparticles with Upconversion Luminescence for Possible Applications in Security. Nanotechnology. 2009; 20(18):185301. [PubMed: 19420608]

20. Wang HQ, Batentschuk M, Osvet A, Pinna L, Brabec CJ. Rare-Earth Ion Doped up-Conversion Materials for Photovoltaic Applications. Adv Mater. 2011; 23(22-23):2675-80. [PubMed: 21823249]

21. Haase M, Schafer H. Upconverting Nanoparticles. Angew Chem Int Edit. 2011; 50(26):58085829.

22. Wang F, Banerjee D, Liu YS, Chen XY, Liu XG. Upconversion Nanoparticles in Biological Labeling, Imaging, and Therapy. Analyst. 2010; 135(8):1839-1854. [PubMed: 20485777]

23. Achatz DE, Ali R, Wolfbeis OS. Luminescent Chemical Sensing, Biosensing, and Screening Using Upconverting Nanoparticles. Top Curr Chem. 2011; 300:29-50. [PubMed: 21516435]

24. Zhou B, Shi B, Jin D, Liu X. Controlling Upconversion Nanocrystals for Emerging Applications. Nat Nanotechnol. 2015; 10(11):924-36. [PubMed: 26530022]

25. Lim X. The Nanolight Revolution Is Coming. Nature. 2016; 531:26-28. [PubMed: 26935679]

26. King RS, Hallett PM, Foster D. Nir-Nir Fluorescence: A New Genre of Fingermark Visualisation Techniques. Forensic Sci Int. 2016; 262:e28-33. [PubMed: 27040305]

27. Wang M, Abbineni G, Clevenger A, Mao C, Xu S. Upconversion Nanoparticles: Synthesis, Surface Modification and Biological Applications. Nanomedicine. 2011; 7(6):710-29. [PubMed: 21419877]

28. Pan AC, del Canizo C, Luque A. Characterization of up-Converter Layers on Bifacial Silicon Solar Cells. Mater Sci Eng B-Adv. 2009; 159-60:212-215.

29. Kobayashi H, Kosaka N, Ogawa M, Morgan NY, Smith PD, Murray CB, Ye XC, Collins J, Kumar GA, Bell H, Choyke PL. In Vivo Multiple Color Lymphatic Imaging Using Upconverting Nanocrystals. J Mater Chem. 2009; 19(36):6481-6484.

30. Maddox PS, Moree B, Canman JC, Salmon ED. Spinning Disk Confocal Microscope System for Rapid High-Resolution, Multimode, Fluorescence Speckle Microscopy and Green Fluorescent Protein Imaging in Living Cells. Methods Enzymol. 2003; 360:597-617. [PubMed: 12622170]

31. Davies T, Jordan SN, Chand V, Sees JA, Laband K, Carvalho AX, Shirasu-Hiza M, Kovar DR, Dumont J, Canman JC. High-Resolution Temporal Analysis Reveals a Functional Timeline for the Molecular Regulation of Cytokinesis. Dev Cell. 2014; 30(2):209-23. [PubMed: 25073157]

32. Pollnau M, Gamelin D, Lüthi S, Güdel H, Hehlen M. Power Dependence of Upconversion Luminescence in Lanthanide and Transition-Metal-Ion Systems. Physical Review B. 2000; 61(5): 3337.

33. Pan AC, del Canizo C, Luque A. Characterization of up-Converter Layers on Bifacial Silicon Solar Cells. Mater Sci Eng B-Adv. 2009; 159-160:212-215.

34. de Wild J, Meijerink A, Rath JK, van Sark WGJHM, Schropp REI. Upconverter Solar Cells: Materials and Applications. Energy and Environmental Science. 2011; 4:4835-4848.

35. Li X, Li Q, Wang J, Li J. Hydrothermal Synthesis of Er-Doped Yttria Nanorods with Enhanced Red Emission Via Upconversion. J Lumin. 2007; 124(2):351-356.

36. Liu G. Advances in the Theoretical Understanding of Photon Upconversion in Rare-Earth Activated Nanophosphors. Chem Soc Rev. 2015; 44(6):1635-1652. [PubMed: 25286989]

37. Jaba N, Kanoun A, Mejri H, Selmi A, Alaya S, Maaref H. Infrared to Visible up-Conversion Study for Erbium-Doped Zinc Tellurite Glasses. Journal of Physics: Condensed Matter. 2000; 12(20): 4523.

38. Zhao J, Lu Z, Yin Y, McRae C, Piper JA, Dawes JM, Jin D, Goldys EM. Upconversion Luminescence with Tunable Lifetime in Nayf 4: Yb, Er Nanocrystals: Role of Nanocrystal Size. Nanoscale. 2013; 5(3):944-952. [PubMed: 23223581] 
39. Sarkar S, Meesaragandla B, Hazra C, Mahalingam V. Sub-5 Nm Ln(3)(+)-Doped Baluf(5) Nanocrystals: A Platform to Realize Upconversion Via Interparticle Energy Transfer (Ipet). Adv Mater. 2013; 25(6):856-60. [PubMed: 23118000]

40. Baeumle, M. Sigma Aldrich Sunstone Luminescent Ucp Nanocrystals Web Resource. 2016. http:// www.sigmaaldrich.com/

41. Corstjens PL, Li S, Zuiderwijk M, Kardos K, Abrams WR, Niedbala RS, Tanke HJ. Infrared upConverting Phosphors for Bioassays. IEE Proc Nanobiotechnol. 2005; 152(2):64-72. [PubMed: 16441160]

42. Zheng X, Krakowiak J, Patel N, Beyzavi A, Ezike J, Khalil AS, Pincus D. Dynamic Control of Hsf1 During Heat Shock by a Chaperone Switch and Phosphorylation. Elife. 2016; 5

43. Brandman O, Stewart-Ornstein J, Wong D, Larson A, Williams CC, Li GW, Zhou S, King D, Shen PS, Weibezahn J, Dunn JG, Rouskin S, Inada T, Frost A, Weissman JS. A Ribosome-Bound Quality Control Complex Triggers Degradation of Nascent Peptides and Signals Translation Stress. Cell. 2012; 151(5):1042-54. [PubMed: 23178123]

44. Anckar J, Sistonen L. Regulation of Hsf1 Function in the Heat Stress Response: Implications in Aging and Disease. Annu Rev Biochem. 2011; 80:1089-115. [PubMed: 21417720]

45. Lambert TJ, Waters JC. Assessing Camera Performance for Quantitative Microscopy. Methods Cell Biol. 2014; 123:35-53. [PubMed: 24974021]

46. Atlas, RM. Handbook of Microbiological Media. 4th. ASM Press; CRC Press/Taylor \& Francis; Washington, D.C. Boca Raton, FL: 2010. p. 2036 p 
A

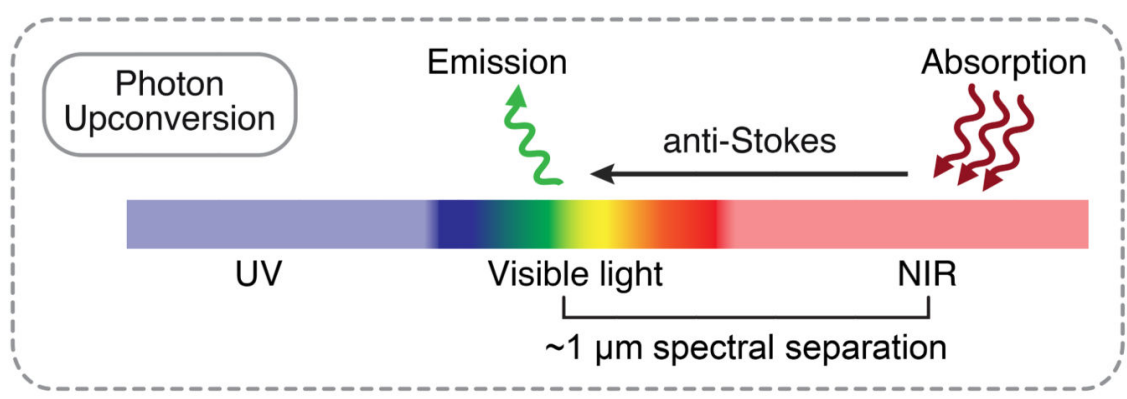

Power dependence for micro ${ }^{540}$-UCPs with $1470 \mathrm{~nm}$ excitation from $4.8-27.5 \times 10^{4}$ Suns on a spinning disc confocal microscope

B

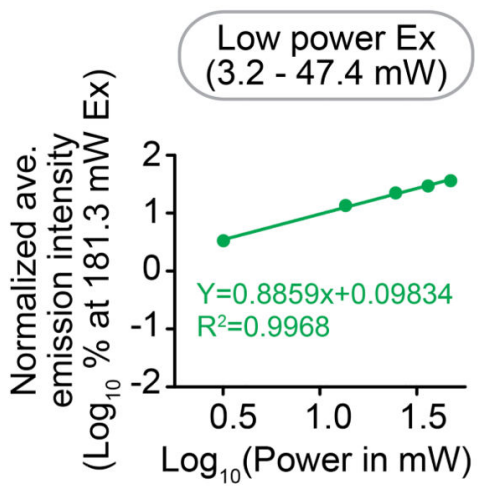

$\mathrm{D}$

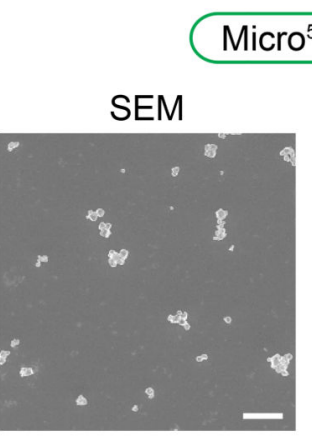

C

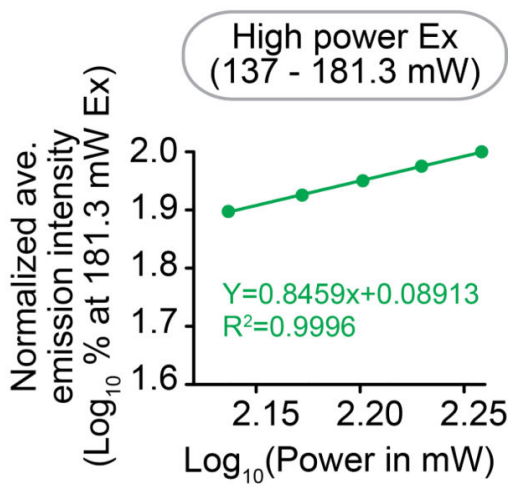

E

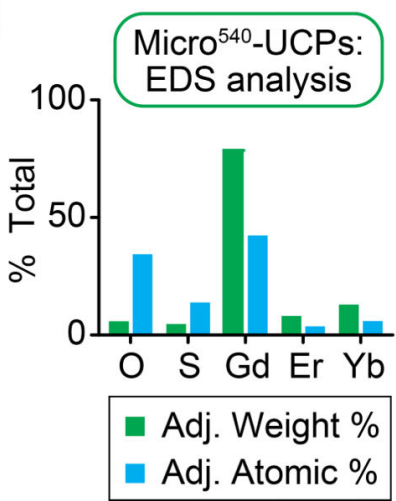

Figure 1. Micro ${ }^{540}$-UCP upconverting particles emit visible light upon excitation with NIR radiation with high efficiency

(A) Schematic representation of the principle of photon upconversion. Upon excitation by infrared radiation, upconverting nanoparticles undergo an anti-Stokes shift phenomenon and emit light in the visible region of the spectrum. (B, C) Log-log plots showing the micro ${ }^{540}$ UCPs' power dependence between average emission intensity and excitation laser power between (B) 3.2-47.4 $\mathrm{mW}$ and (C) 137-181.3 mW measured within an ROI (normalized to the average intensity within an ROI at $181.3 \mathrm{~mW}$ laser excitation). Slopes decreased with increasing laser power. The change in power dependence was found to be from $\mathrm{P}^{0.89}$ to $\mathrm{P}^{0.85}$ for micro ${ }^{540}$-UCPs. Data represents mean from $\mathrm{n}>5$ independent experiments. (D) 
Representative SEM image and particle size distribution histogram of micro ${ }^{540}$-UCPs. Scale bar $=10 \mu \mathrm{m}$. (E) EDS analysis of micro-UCPs. 
A

B

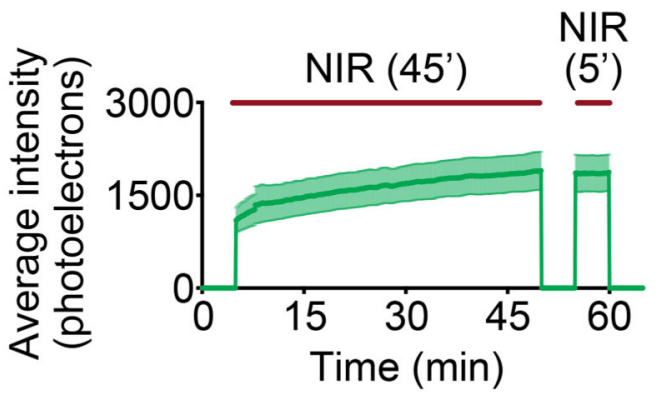

\section{Photostability of micro ${ }^{540}$-UCPs:} $1470 \mathrm{~nm}$ Ex 525/50 nm Em
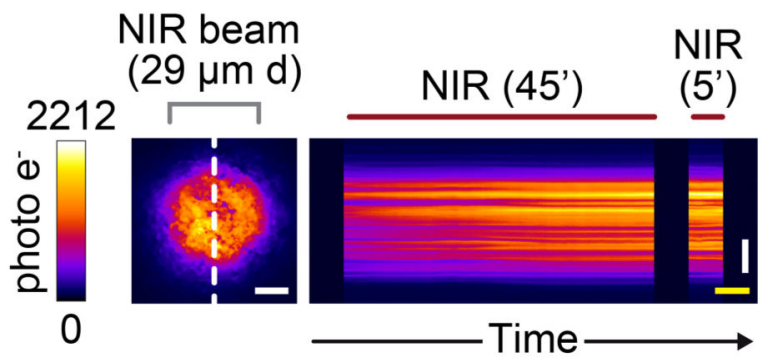

C

\section{Response time of micro ${ }^{540}-U C P s:$} $1470 \mathrm{~nm}$ Ex 525/50 nm Em

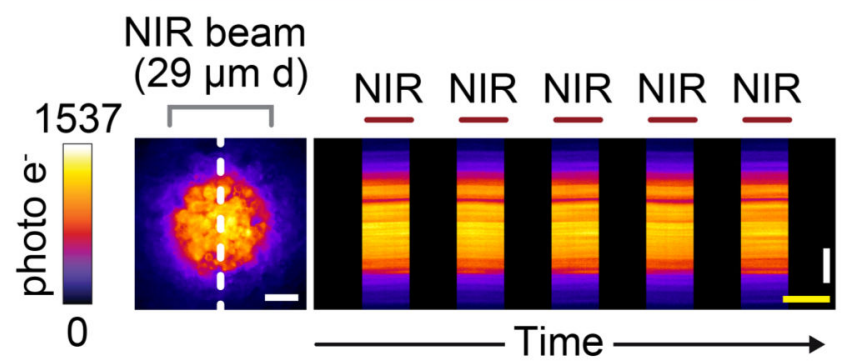

$E$

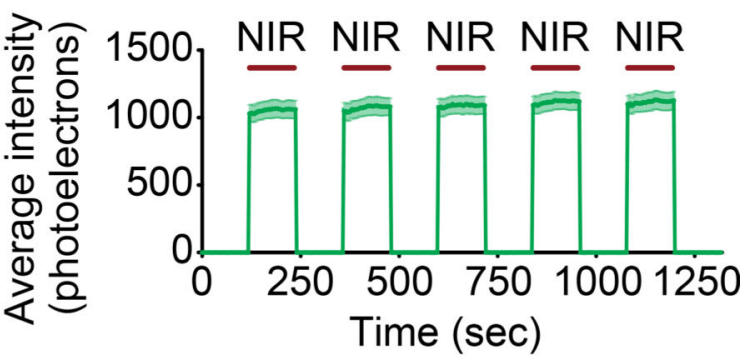

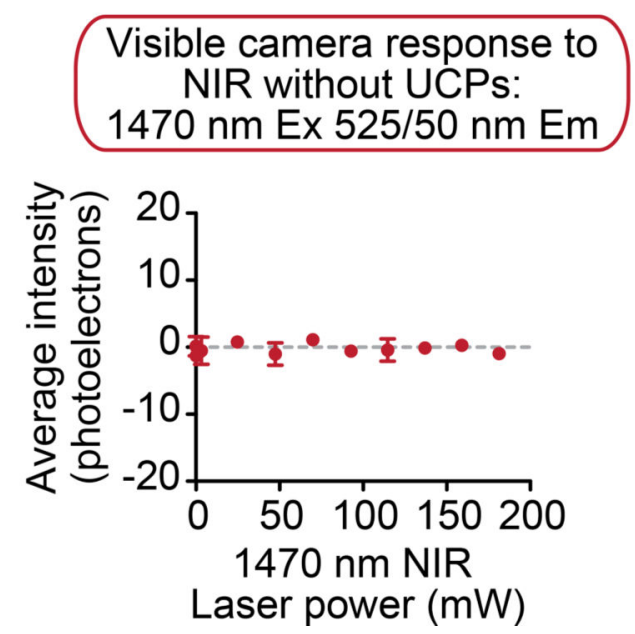

Figure 2. Micro ${ }^{540}$-UCPs are robustly photo-stable and respond rapidly to excitation light Kymographs generated from the time lapse imaging of (A) micro ${ }^{540}$-UCPs to demonstrate the emission photostability after a 45-min period of continuous irradiation with $1470 \mathrm{~nm}$ NIR light. For (A, B) this initial irradiation was followed by a 5 min off period, and then another 5 min NIR on period to show the emission intensity returns to the highest level with micro ${ }^{540}$-UCPs. (C, D) Kymograph and quantitative visible emission intensity analysis (average minus background) of the visible emission light generated over time for micro ${ }^{540}$ UCPs to demonstrate the visible light emission response speed to 2-min on and off pulses of $1470 \mathrm{~nm}$ irradiation. (A, C) The white dashed line indicates the approximate region used for plotting the kymographs. (A-D) The duration of NIR irradiation is indicated by the maroon 
bars on top of the kymographs. FIRE LUT used to indicate emission intensity. Scale bars (white $=10 \mu \mathrm{m})$ and (yellow $=2 \mathrm{~min})$. Data represents mean \pm standard error from $\mathrm{n}=3(\mathrm{~A}$, B) or $\mathrm{n}=5(\mathrm{C}, \mathrm{D})$ independent experiments. (E) Control, background signal quantification showing the lack of detectable emission intensity on the visible light sensitive camera upon excitation with increasing power levels of $1470 \mathrm{~nm}$ light through a $525 \pm 25 \mathrm{~nm}$ emission filter in the absence of UCPs. 


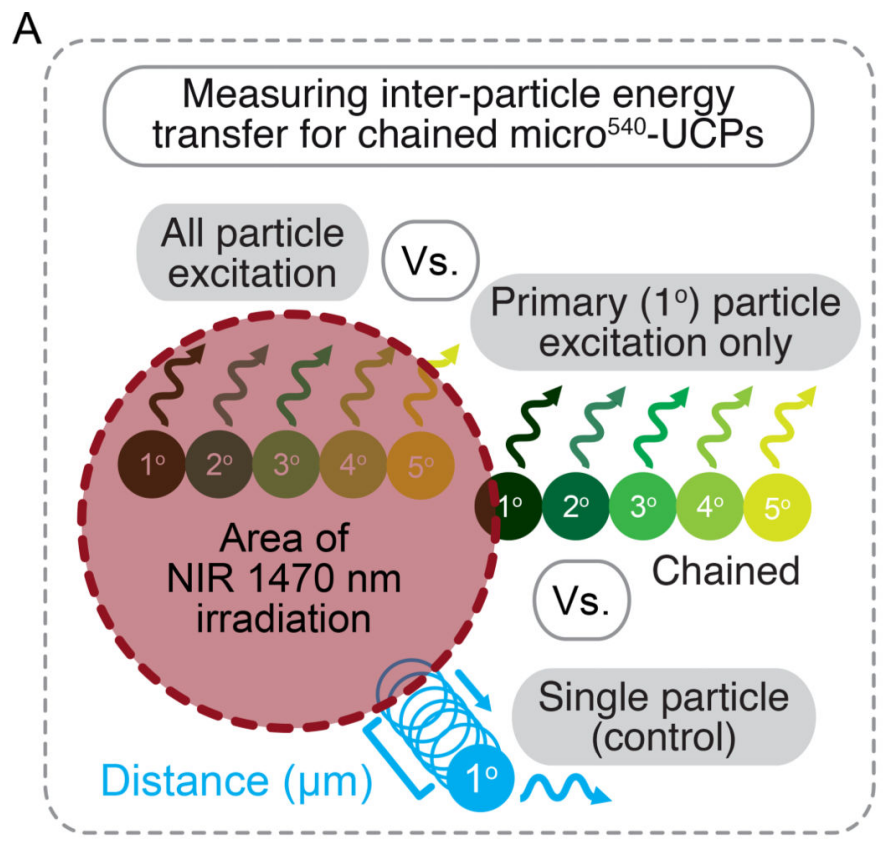

B

C
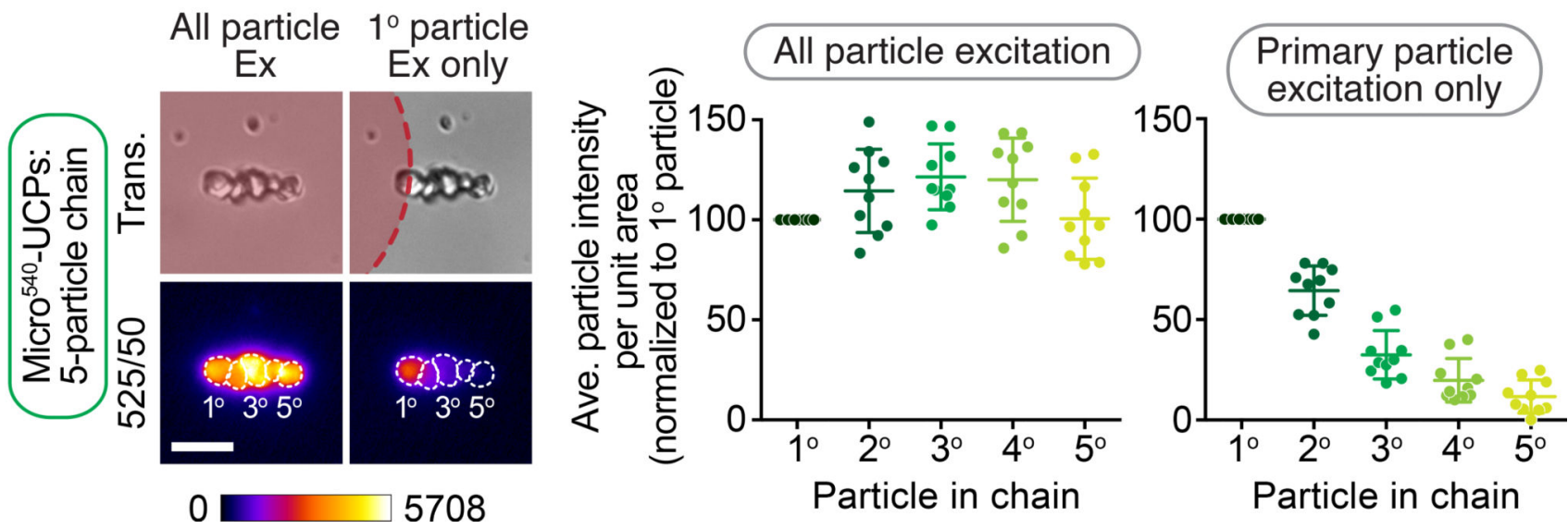

Figure 3. Micro ${ }^{540}$-UCPs show significant particle-to-particle energy transfer

(A) Schematic representation of the experimental setup to test the NIR laser spreading profile and inter-particle energy transfer of micro ${ }^{540}$-UCPs. To test the NIR laser spreading, a single micro ${ }^{540}$-UCP (blue) was irradiated by the NIR beam and imaged while moving the microscope stage in $\sim 1 \mu \mathrm{m}$ increments away from the NIR beam per acquisition interval. To test inter-particle energy transfer, the NIR laser was used to irradiate either all particles in a chain of five $\left(1^{\circ}-5^{\circ}\right)$ or just the primary particle $\left(1^{\circ}\right)$ in the chain. $(\mathrm{C})$ Representative images of a chain of 5 micro ${ }^{540}$-UCPs where either all 5 (left panels) or just the $1^{\circ}$ particle (right panels) are excited by the NIR laser. White dashed lines represent the edges of individual microparticles in the chain as seen in the transmitted light image used for the ROI for quantification in (B, D). FIRE LUT used to indicate emission intensity. Scale bars $=5 \mu \mathrm{m}$ (B) Scatter plot of sum projected average particle intensity within an ROI roughly equal to the area of individual micro ${ }^{540}$-UCPs particles within either a single particle (blue) or chains of 5 particles (green) versus distance from the edge of the NIR beam, $n=10$ individual and 
chains of particles. Curve fitting was done using a one phase decay equation (D) Quantification of sum projected average particle intensity within an ROI roughly equal to the area of individual micro ${ }^{540}$-UCPs normalized to $1^{\circ}$ particle for each of the 5 microparticles in a chain when either all 5 particles or just the $1^{\circ}$ particle are/is excited by the NIR laser, $\mathrm{n}=10$ chains of particles. 
A

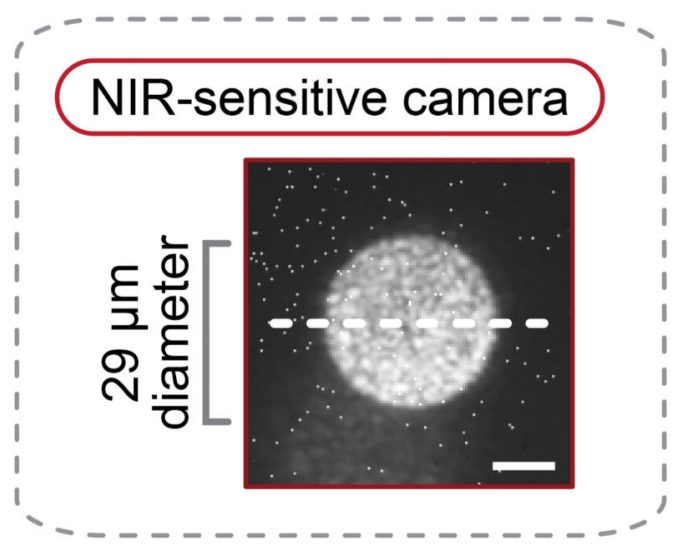

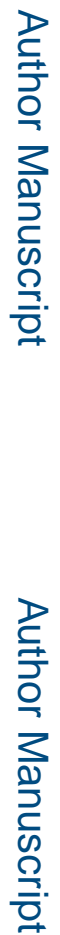

C

Micro ${ }^{540}-U C P S$

In focus

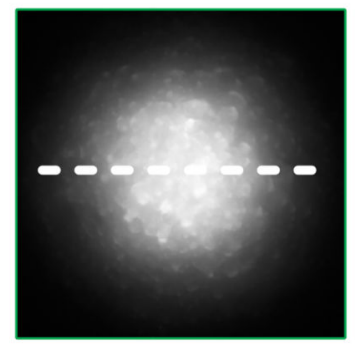

Out of focus
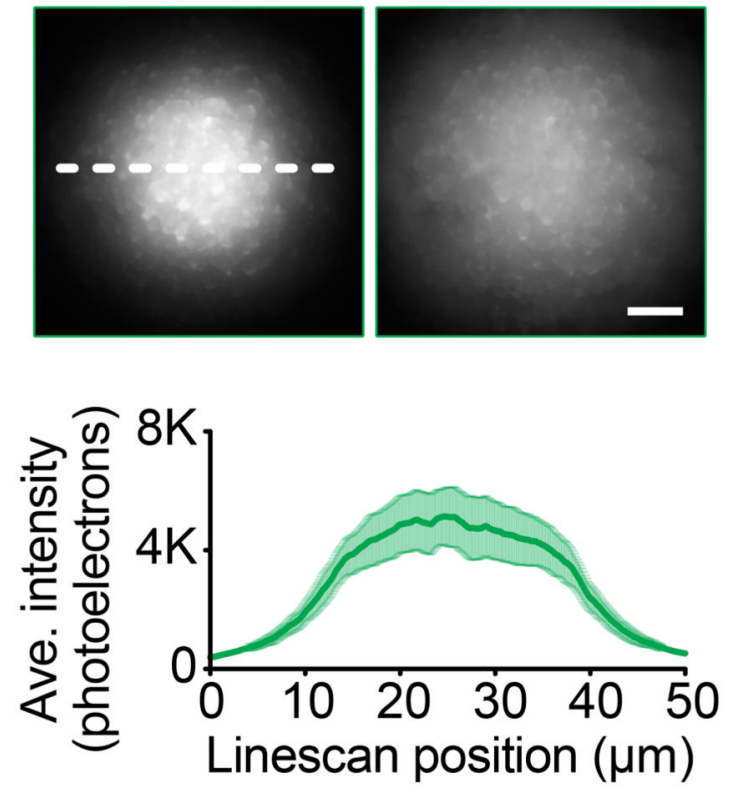

$\mathrm{B}$

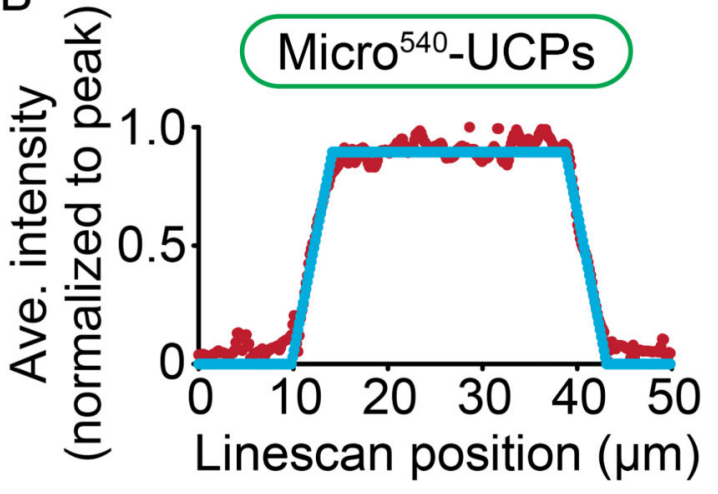

- Power scaled NIR profile - Ideal laser fit

D

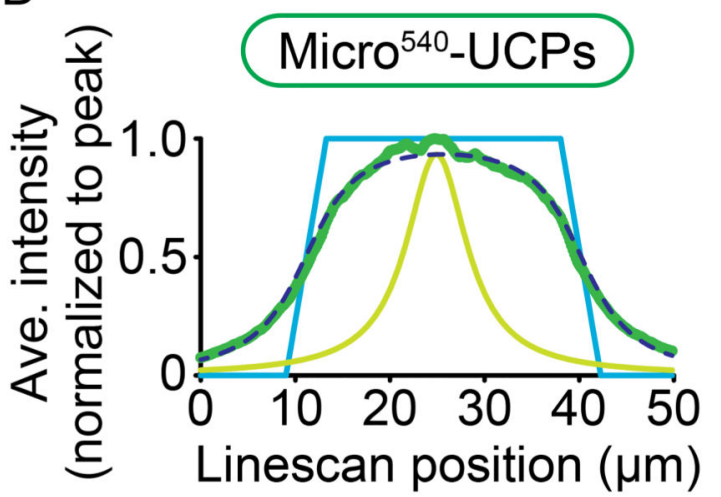

- Measured emission profile

- Ideal laser fit

-- Fitted model

- Probability Density Function (PDF)

Figure 4. Beam profiles from micro ${ }^{540}$-UCPs show a broad Lorentzian fit

Image (A) of $29 \mu \mathrm{m}$ diameter masked circular region of $1470 \mathrm{~nm}$ NIR irradiation on a mirrored slide taken with an NIR-sensitive camera. (B) The beam profile of the excitation laser (shown in maroon) is recorded and then adjusted to the power dependence for micro ${ }^{540}$-UCPs (Figure 1C) and finally, these profiles are fitted to an ideal trapezoidal laser profile (shown in Carolina blue) to remove the noise. (C) Representative images of the visible light emission from micro ${ }^{540}$-UCPs irradiated with a $29 \mu \mathrm{m}$ diameter NIR beam when the beam is either in focus (left panel) or out of focus (right panel) at the imaging plane. Scale bars $=10 \mu \mathrm{m}$. Background subtracted line scans of the in-focus images in (C) 
were performed and plotted as shown (C). The white dashed lines across the images indicate the region used for plotting the line scans. (D) Final result of fitting the beam profiles for micro ${ }^{54}$-UCPs (in green) to the convolution of the ideal laser profiles (in Carolina blue; B, D) and a Lorentzian probability density function (in light green), with the result of the best fit plotted with a dashed dark blue line (D). Data represents mean \pm standard error from $n=$ 3 independent experiments. 


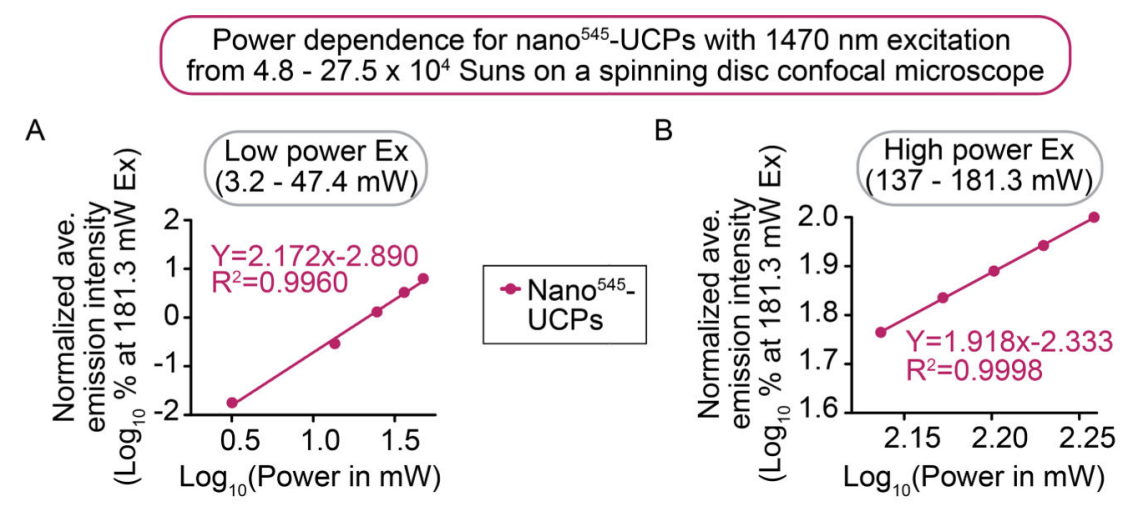

C

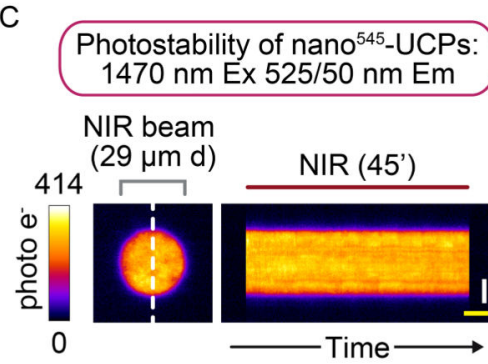

$\mathrm{E}$

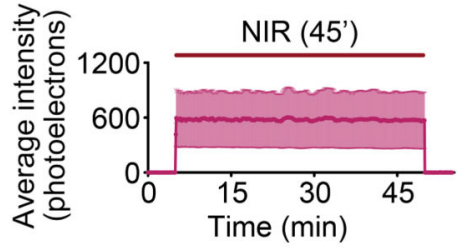

G
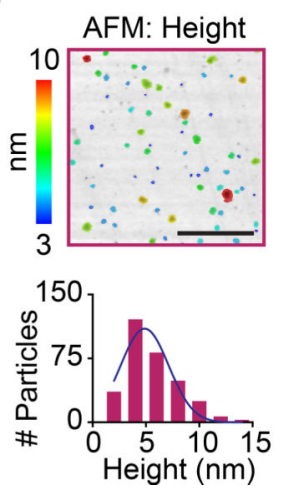

$\mathrm{H}$
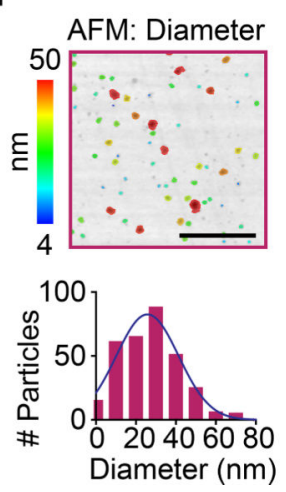

D

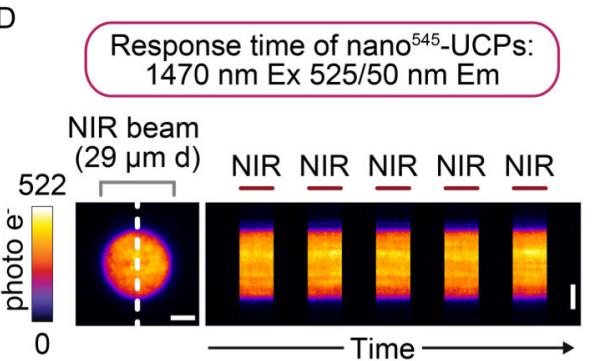

F

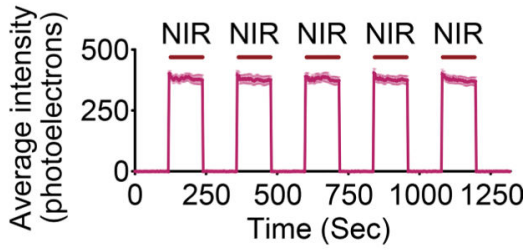

I

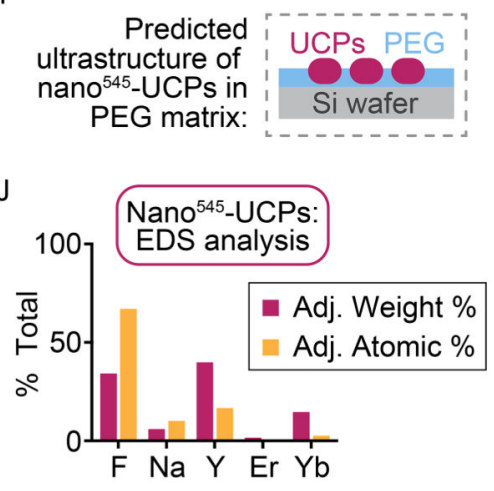

Figure 5. Nano $^{545}$-UCPs emit visible light upon excitation with NIR radiation with modest efficiency

(A, B) Log-log plots showing the power dependence for nano ${ }^{545}$-UCPs between average emission intensity and excitation laser power between (B) 3.2-47.4 $\mathrm{mW}$ and (C) 137-181.3 $\mathrm{mW}$ measured within an ROI (normalized to the average intensity within an ROI at 181.3 $\mathrm{mW}$ laser excitation). Slopes decreased with increasing laser power. The change in power dependence was found to be from $\mathrm{P}^{2.17}$ to $\mathrm{P}^{1.92}$ for nano ${ }^{545}$-UCPs. Data represents mean from $n>5$ independent experiments. (C) Kymograph generated and (E) quantitative visible emission intensity analysis from time lapse imaging of nano ${ }^{545}$-UCPs after a 45 -min period of continuous irradiation with $1470 \mathrm{~nm}$ NIR light. (D) Kymograph and (F) quantitative 
visible emission intensity analysis (average minus background) of the visible emission light generated over time for nano ${ }^{545}$-UCPs to demonstrate the visible light emission response speed to five 2-min on and off pulses of $1470 \mathrm{~nm}$ irradiation. (C, D) The white dashed line indicates the approximate region used for plotting the kymographs. Scale bars (white $=10$ $\mu \mathrm{m})$ and (yellow $=2 \mathrm{~min})$. $(\mathrm{C}-\mathrm{F})$ The duration of NIR irradiation is indicated by the maroon bars on top of the kymographs. FIRE LUT used to indicate emission intensity. Data represents mean \pm standard error from $n=3(A-C, E)$ or $n=5(D, F)$ independent experiments. $(\mathrm{G}, \mathrm{H})$ AFM images and particle size distribution histograms depicting the $(\mathrm{G})$ height and $(\mathrm{H})$ diameter of nano ${ }^{545}$-UCPs. Black scale bars $=500 \mathrm{~nm}$ ). (I) Schematic demonstrating the nano ${ }^{545}$-UCPs embedded in a PEG matrix during AFM analysis. (J) EDS analysis of nano ${ }^{545}$-UCPs. 
A

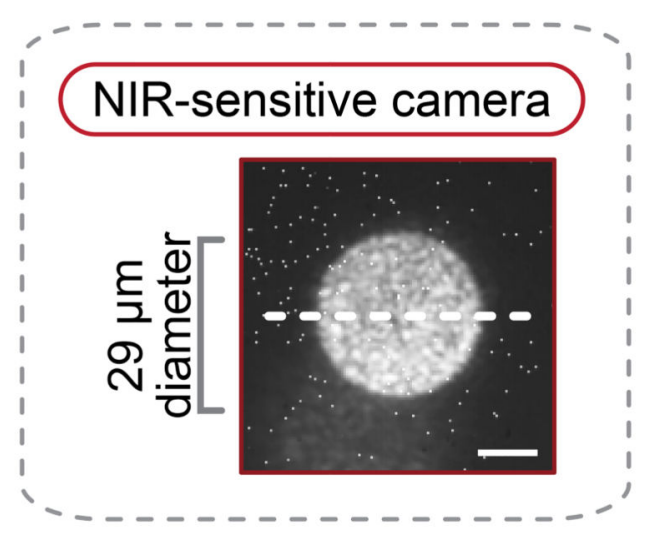

C
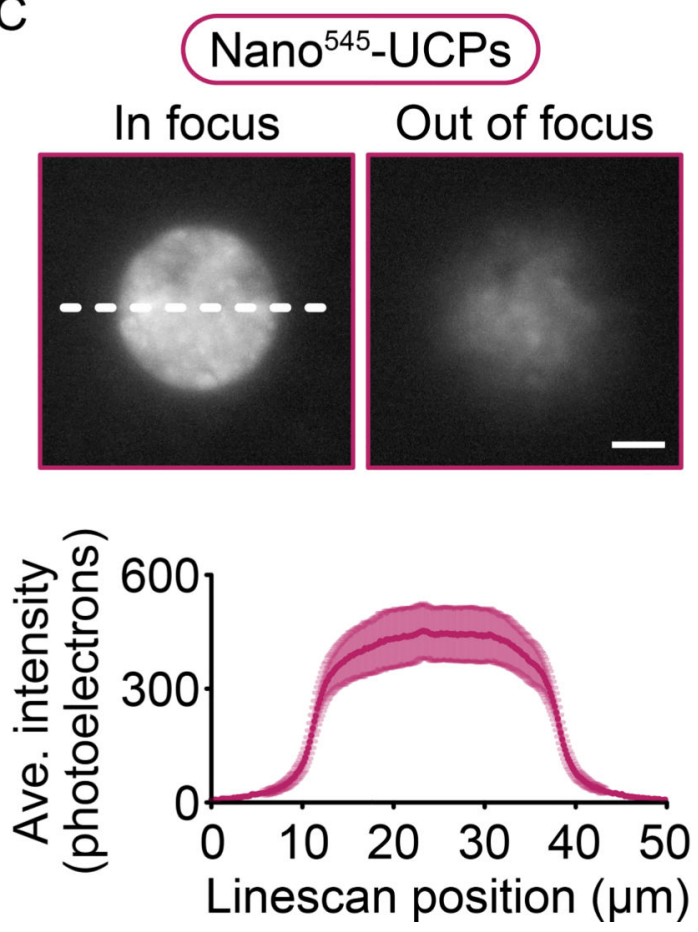

B

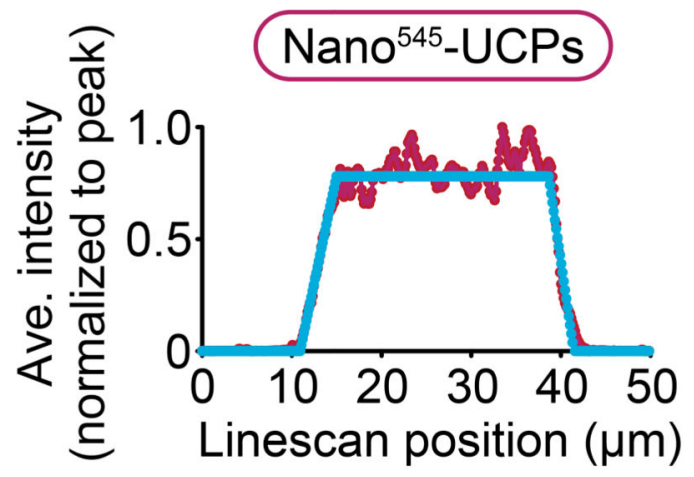

- Power scaled NIR profile

- Ideal laser fit

D

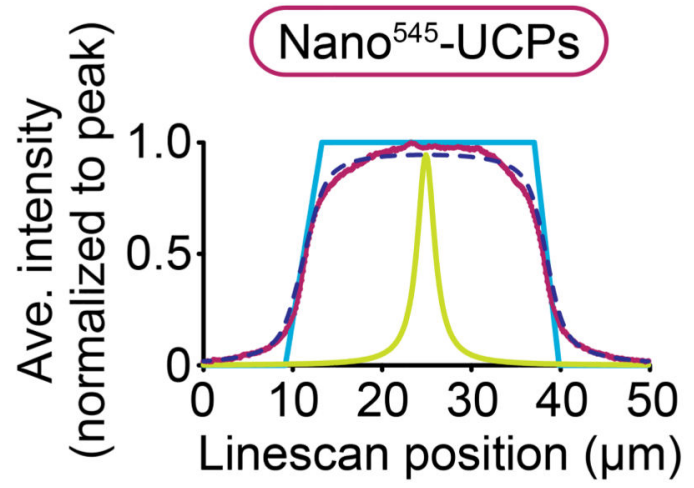

- Measured emission profile - Ideal laser fit

-- Fitted model

- Probability Density

Function (PDF)

Figure 6. Beam profiles from nano ${ }^{545}$-UCPs show a tight Lorentzian fit

Image (A) of $29 \mu \mathrm{m}$ diameter masked circular region of $1470 \mathrm{~nm}$ NIR irradiation on a mirrored slide taken with an NIR-sensitive camera. (B) The beam profile of the excitation laser (shown in maroon) is recorded then adjusted to the power dependence for nano ${ }^{545}$ UCPs (Figure 5B) and these profiles are fitted to an ideal trapezoidal laser profile (shown in Carolina blue) to remove the noise. (C) Representative images of the visible light emission from nano ${ }^{545}$-UCPs irradiated with a $29 \mu \mathrm{m}$ diameter NIR beam when the beam is either in focus (left panel) or out of focus (right panel) at the imaging plane. Scale bars $=10 \mu \mathrm{m}$. Background-subtracted linescans of the in focus images in $(\mathrm{A}, \mathrm{C})$ were performed and 
plotted as shown (C). The white dashed lines across the images indicate the region used for plotting the linescans. (D) Final result of fitting the beam profiles for nano ${ }^{545}$-UCPs (in bright pink) to the convolution of the ideal laser profiles (in Carolina blue; B, D)) and a Lorentzian probability density function (in light green), with the result of the best fit plotted with a dashed dark blue line (D). Data represents mean \pm standard error from $n=3$ independent experiments. 
A
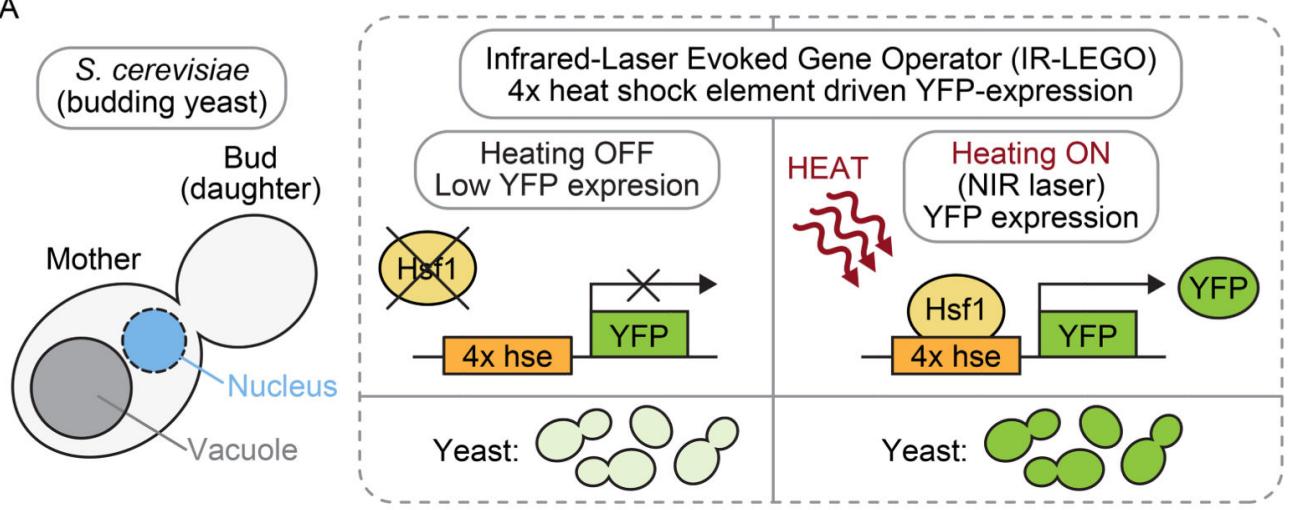

B

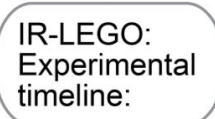

(1) Align NIR to specimen plane using: timeline:

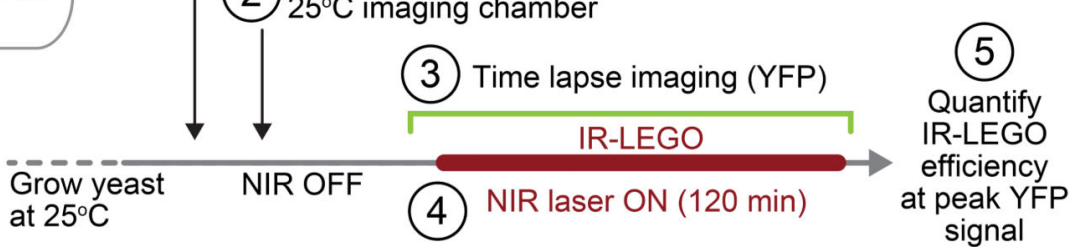

C

Before IR-LEGO After IR-LEGO

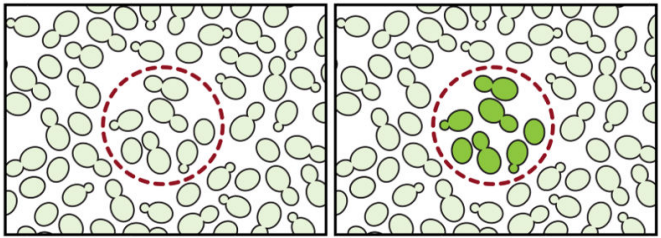

D

$4 \mathrm{x}$ hse::YFP

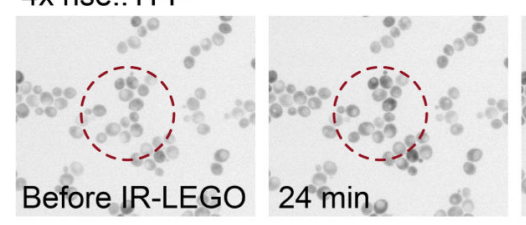

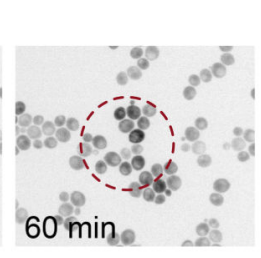

IR-LEGO efficiency (Ave.YFP intensity ratio):

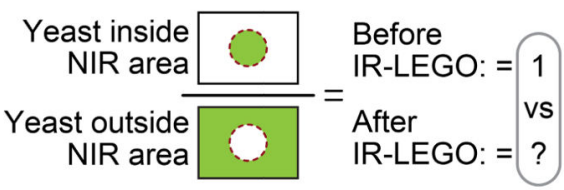

E
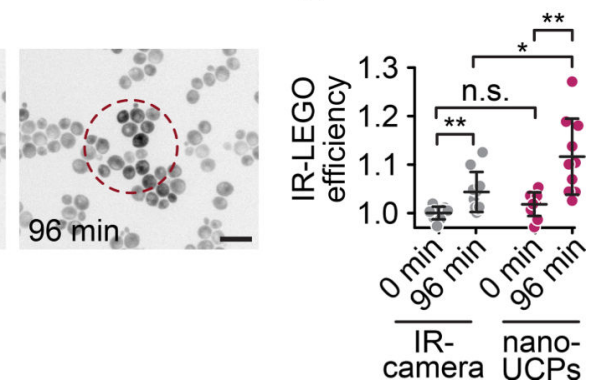

Figure 7. NIR alignment with nano ${ }^{545}$-UCPs improves IR-LEGO efficiency in S. cerevisiae (A) Schematic of a single budding $S$. cerevisiae showing (left panel) the nucleus (blue) and vacuole (grey); schematic depicting the mechanism of the Infrared-Laser-Evoked Gene Operator (IR-LEGO) assay (right panel). Strain DPY144 contains a genetically encoded 4x repeated heat shock element $(4 \times h s e)$ driving Yellow Fluorescent Protein (YFP). At normal growth temperature $\left(25-30^{\circ} \mathrm{C}\right)$, YFP is not expressed, but upon local heating to a higher temperature through use of a $1470 \mathrm{~nm}$ NIR laser, the heat-sensitive transcription factor Heat Shock Factor 1 (Hsf1) induces YFP expression. B) Experimental timeline for IR-LEGO experiment depicting the individual steps in the experiment. C) A $29 \mu \mathrm{m}$ mask was used to 
delimit the area of NIR irradiation to a sub-set of yeast cells (red dashed outline) within the field of view. IR-LEGO efficiency was calculated based on average YFP signal intensity per yeast cell within the irradiated area divided by the average YFP signal intensity per yeast cell outside of the irradiated area. D) Representative images of yeast showing increased YFP expression within the NIR-irradiated region over time (red dashed line), and little change outside of the NIR-irradiated region. Scale bar $=10 \mu \mathrm{m}$. E) IR-LEGO efficiency is significantly higher when nano ${ }^{545}$-UCPs were used to align the $1470 \mathrm{~nm}$ laser to the imaging plane relative to when a second NIR-sensitive camera was used for alignment $(\mathrm{p}=$ $0.0161, *)$. IR-LEGO efficiency at peak time (96 $\mathrm{min}$ ) when using nano ${ }^{545}$-UCPs based NIR alignment is 1.117 ( $11.7 \%$ increase; $\mathrm{n}=10 ; \mathrm{p}=0.0013$, ** relative to pre-IR-LEGO), whereas IR-LEGO efficiency when using NIR-sensitive camera based NIR alignment is 1.044 ( $4.4 \%$ increase; $\mathrm{n}=10 ; \mathrm{p}=0.0053$, ** relative to pre-IR-LEGO). There is no significant difference in pre-IR-LEGO levels between the two methods $(\mathrm{p}=0.0585$, n.s.). 